\title{
1 An association between the gut microbiota and immune cell dynamics in humans.
}

2 Jonas Schluter ${ }^{1}$, Jonathan U. Peled ${ }^{2,3}$, Bradford P. Taylor ${ }^{1}$, Melody Smith ${ }^{2,3}$, Kate A. Markey ${ }^{2,3}$, Ying

3 Taur $^{4}$, Rene Niehus ${ }^{5}$, Anna Staffas ${ }^{6}$, Anqi Dai ${ }^{2}$, Emily Fontana ${ }^{4}$, Luigi A. Amoretti ${ }^{4}$, Roberta J.

4 Wright $^{4}$, Sejal Morjaria ${ }^{4}$, Maly Fenelus ${ }^{7}$, Melissa S. Pessin ${ }^{7}$, Nelson J. Chao ${ }^{8}$, Meagan Lew ${ }^{8}$, Lauren

5 Bohannon ${ }^{8}$, Amy Bush $^{8}$, Anthony D. Sung ${ }^{8}$, Tobias M. Hohl ${ }^{4}$, Miguel-Angel Perales ${ }^{2,3}$, Marcel R.M.

6 van den Brink ${ }^{2,3}$, Joao B. Xavier ${ }^{1}$

7 1) Computational and Systems Biology Program, Sloan Kettering Institute, Memorial Sloan Kettering Cancer

8 Center, New York, NY, USA

9 2) Adult Bone Marrow Transplantation Service, Department of Medicine, Memorial Sloan Kettering Cancer

10 Center, New York, NY, USA

11 3) Weill Cornell Medical College, New York, NY, USA

12 4) Infectious Disease Service, Department of Medicine, and Immunology Program, Sloan Kettering Institute,

13 New York, NY, USA.

14 5) Harvard University, T.H. Chan School of Public Health, 677 Huntington Avenue, Boston, MA 02115

15 6) Sahlgrenska Cancer Center, Department of Microbiology and Immunology, Institute of Biomedicine,

16 University of Gothenburg, SE-405 30, Gothenburg, Sweden

17 7) Department of Laboratory Medicine, Memorial Sloan Kettering Cancer Center, New York, NY, USA

18 8) Division of Hematologic Malignancies and Cellular Therapy, Duke University School of Medicine

19 Correspondence: jonas.schluter+mskcc@gmail.com, xavierj@mskcc.org 


\section{ABSTRACT}

22 The gut microbiota influences development and homeostasis of the mammalian immune system ${ }^{1-3}$,

23 can alter immune cell compositions in mice $^{4-7}$, and is associated with responses to immunotherapy

24 that rely on the activity of peripheral immune cells ${ }^{8-12}$. Still, our understanding of how the microbiota

25 modulates immune cells dynamics remains limited, particularly in humans where a lack of deliberate

26 manipulations makes inference challenging. Here we study hundreds of hospitalized—and closely

27 monitored - patients receiving hematopoietic cell transplantation as they recover from chemotherapy

28 and stem cell engraftment. This aggressive treatment causes large shifts in both circulatory immune

29 cell and microbiota populations, allowing the relationships between the two to be studied

30 simultaneously. We analyzed daily changes in white blood cells from 2,235 patients, and 10,680

31 longitudinal microbiota samples to identify bacteria associated with those changes. Bayesian

32 inference and validation across patient cohorts revealed consistent associations between gut bacteria

33 and white blood cell dynamics in the context of immunomodulatory medications, clinical metadata

34 and homeostatic feedbacks. We contrasted the potency of fermentatively active, obligate anaerobic

35 bacteria with that of medications with known immunomodulatory mechanism to estimate the potential

36 of the microbiota to influence peripheral immune cell dynamics. Our analysis establishes and

37 quantifies the link between the gut microbiota and the human immune system, with implications for

38 microbiota-driven modulation of immunity. 
40 Experiments in mice provide evidence that the mammalian intestinal microbiome influences the

41 development ${ }^{1-3}$ and homeostasis of its host's immune system ${ }^{4-7,13-15}$. In humans, inflammatory bowel

42 diseases correlate with functional dysbiosis in the gut microbiota ${ }^{16,17}$. Children born preterm and at

43 term have different gut microbiome compositions and differ in the development of immune cell

44 populations in their blood ${ }^{18}$. The composition of the gut microbiota may also influence the success of immunotherapies $^{8-11}$. Immune checkpoint inhibitor therapy relies on activation of circulating T-cells and its success has, independently, been associated with abundances of intestinal anaerobic genera such as Akkermansia $^{9}$ and Faecalibacterium ${ }^{10}$. It is therefore an intriguing prospect to augment treatments such as cancer immunotherapy ${ }^{19}$, including the burgeoning field of chimeric antigen receptor (CAR) T-cell therapy ${ }^{20}$, by leveraging microbiome-driven immune system modulation. humans, and how this compares to deliberate immunomodulatory interventions nevertheless remains limited. Experiments with animals may not always be sufficient to study mechanisms of microbiome-immune interactions and translate them to human biology as the microbial ecology in the gut of an animal model may be different from humans receiving treatment ${ }^{21}$. On the other hand, studies directly in patients may be criticized when they have small subject numbers, are crosssectional, lack statistical power, or disregard key confounders such as medications ${ }^{21}$. To overcome these limitations, we conducted a large-scale longitudinal study of the gut microbiota and day-by-day changes in circulatory immune cell counts. We investigated immune reconstitution dynamics after allogeneic hematopoietic cell therapy (HCT) within all 2,926 patients who underwent HCT at Memorial Sloan Kettering for various hematological malignancies, including leukemia, between 2003 and 2019 (Figure 1A, Table S1). The conditioning regimen of radiation and chemotherapy administered to HCT patients is the most severe perturbation to the immune system deliberately performed in humans and thus offers a unique opportunity to investigate dynamic links between the gut microbiota and the immune system directly in humans. Conditioning depletes white blood cell counts (Figure 1A) and can lead to prolonged periods of neutropenia ( $<500$ neutrophils per $\mu$ blood). Immune reconstitution begins after transplanted stem cells have matured sufficiently to 

with $>500$ neutrophils per $\mu$ l blood, Figure 1A-C). The blood of each patient is carefully monitored

A)
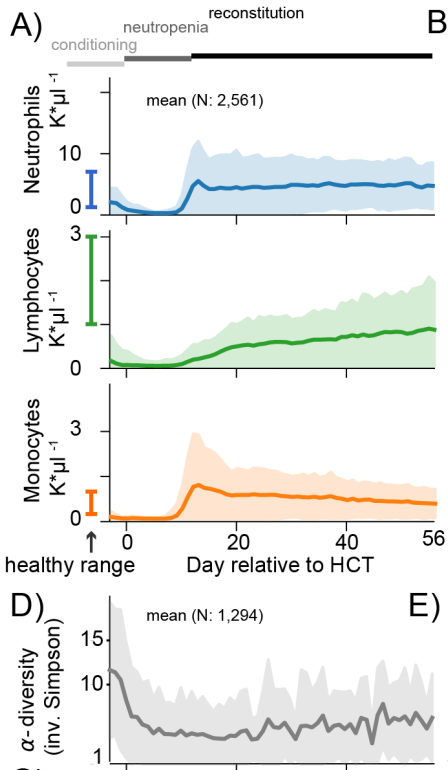

G)
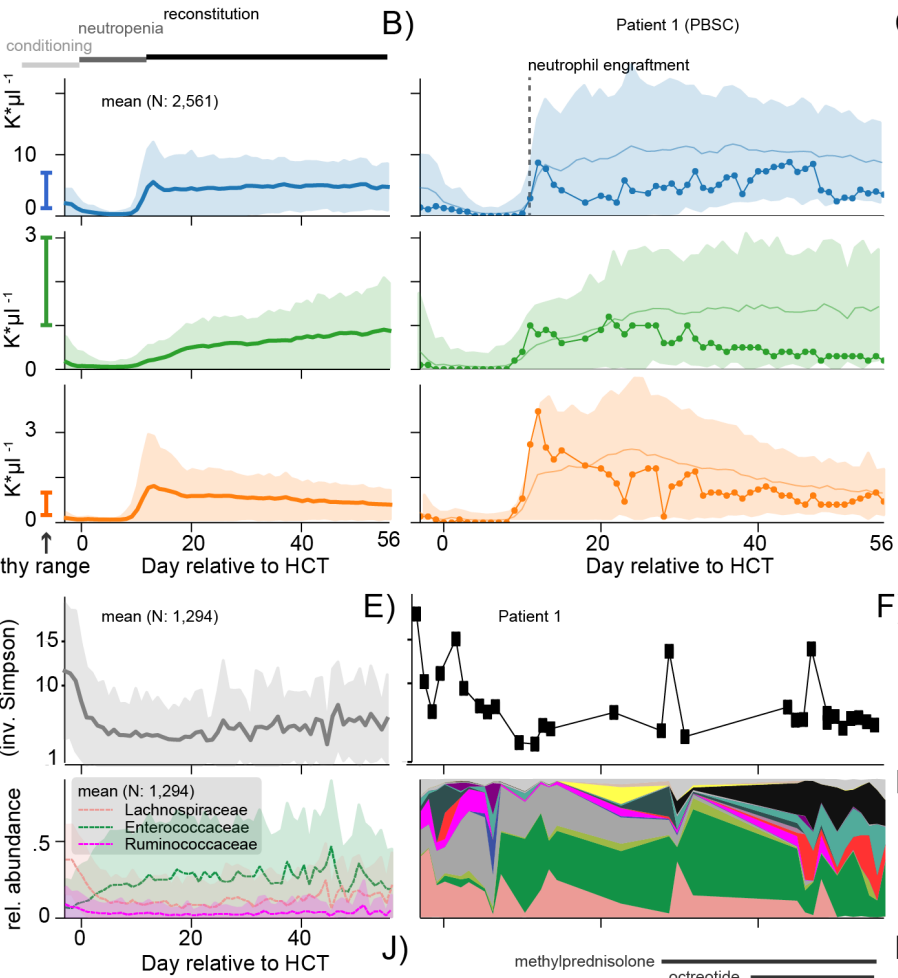

E)
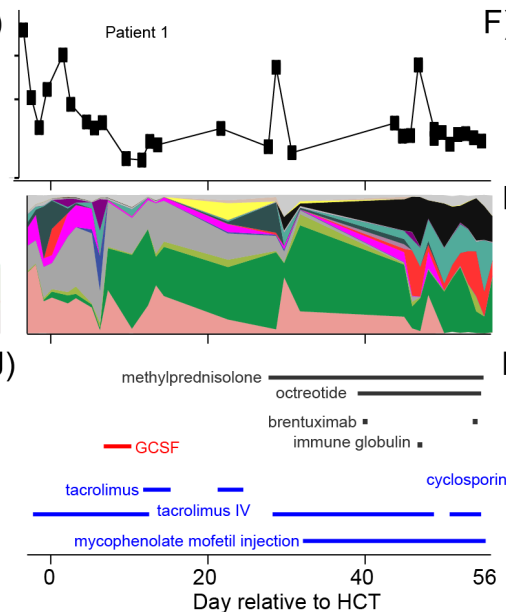

)
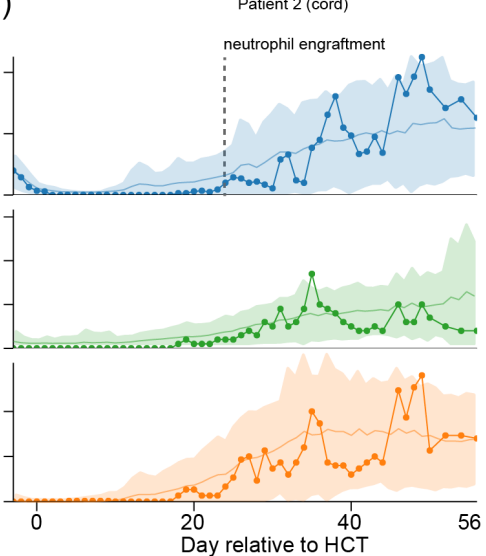

F)

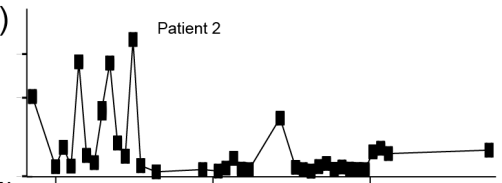

l)

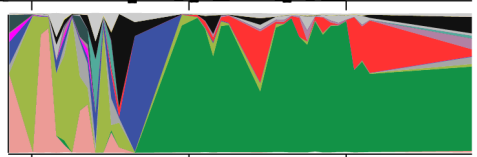

K)

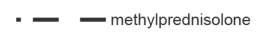

prednisone -

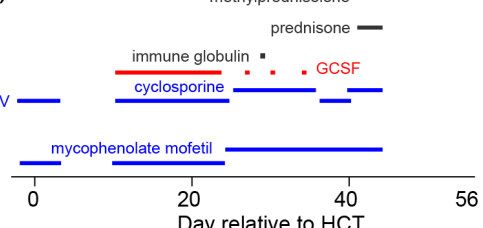

Figure 1: Immune reconstitution and microbiome dynamics after allogeneic hematopoietic cell transplantation (HCT). A) Three major periods of HCT-immunoablation during chemotherapeutic conditioning before HCT, defined as day 0 , post-HCT neutropenia, and reconstitution following neutrophil engraftment-lead to recovery trajectories with large variability between patients. Shown are the mean counts (shaded: \pm 1 standard deviation, $\sigma$ ) of neutrophils, lymphocytes and monocytes per day relative to HCT from patients transplanted between 2003 and 2019 (A), contrasted with two individual patients (B,C) representative of the recovery trajectories for different stem cell graft source; patient 1 who received a peripheral blood stem cell graft, PBSC (line with circles: patient data, solid line and shaded region: mean \pm 1 standard deviation of all PBSC patients), and patient 2 who received a graft of umbilical cord blood (line with circles: patient data, solid line and shaded region: mean \pm 1 standard deviation of all cord patients). Fecal samples collected and analyzed by $16 \mathrm{~S}$ rRNA gene sequencing reveal the loss of microbial diversity reported previously ${ }^{23,24}$ (D, line: mean per day, shaded: \pm 1 standard deviation); E,F: individual patient measurements) and commensal families (G, line: mean relative abundances of bacterial families, shaded: \pm 1 standard deviation); H,I: individual patient measurements), often replaced by Enterococcaceae domination. J,K). Administration of immunomodulatory medications for the two example patients.

throughout this recovery, and medications are administered to modulate the immune cell dynamics,

graft-vs-host disease (Figure $\mathbf{1} \mathbf{J , K}$ ). To investigate if the composition of the gut microbiota is 
metadata of our patients between 3 days before HCT and until 100 days post neutrophil engraftment (excluding pediatric patients, and other exclusion criteria: $\mathrm{N}=2,235$, supplementary methods, Figure

S1). During this period patients are monitored carefully, and our analysis included over 140,000 host phenotype measurements in the form of complete blood counts which quantify the most abundant white blood cells—neutrophils, lymphocytes, monocytes, eosinophils—as well as platelet counts

(Figure 1, S1). We started collecting patients' fecal microbiota data in $2009^{22}$, and by now obtained 10,680 high frequency, longitudinal microbiota compositions.

HCT patients lose gut microbiota biodiversity and commensal microbial families during their treatment (Figure 1D-I); this figure generated from N=1,294 HCT patients clarifies the preliminary trends observed in previous studies from smaller datasets ${ }^{23,24}$. We have shown recently that mice with a depleted intestinal flora had worse recoveries of white blood cells after bone-marrow transplantation ${ }^{25}$. In our patients, microbial diversity usually recovers slowly during white blood cell reconstitution (Figure 1D); however, microbiota recovery as well as immune reconstitution can vary strongly between patients and treatment types (Figure 1B,C,J,K, S1). This variation is illustrated by the distinct trajectories of patient 1 who received a graft of peripheral blood stem cells (PBSC), retained high microbiota diversity and engrafted earlier (Figure 1B,E,H), and patient 2 who received a graft of umbilical cord blood (cord), lost microbiota diversity and engrafted later (Figure 1C,F,I). Low microbiota diversity at the time of neutrophil engraftment has been associated with 5 -fold increased transplant-related mortality ${ }^{26}$, suggesting that that the joint recovery of the microbiota and white blood cells in circulation is critical for clinical outcomes.

To detect a directional and causal link between the microbiota and white blood cells, we first used data from a recent prospective randomized trial of autologous fecal microbiota transplantation (auto-FMT), which is a microbiota manipulation experiment done directly in our patients ${ }^{23}$

97 (supplementary methods). Twenty-four patients (Figure 2A, Table S2) underwent randomization, resulting in 10 untreated control and 14 treated patients, including patient 3 in Figure S2. To investigate if auto-FMT affected white blood cell reconstitution, we compared the 24 patients'

100 neutrophil, lymphocyte, monocyte (Figure 2) and total white blood cell counts (Figure S3) post-

101 engraftment (i.e. when the transplanted hematopoietic cells begin producing new white blood cells, 
102 Figure 2A,S3). FMT procedures were conducted at variable time points relative to neutrophil

103 engraftment, but overall, we observed higher counts of each white blood cell type in patients who

104 received an auto-FMT during the first 100 days post neutrophil engraftment $(\mathrm{p}<0.001$, Figure 2B,S3-

105 S6).

106

A)
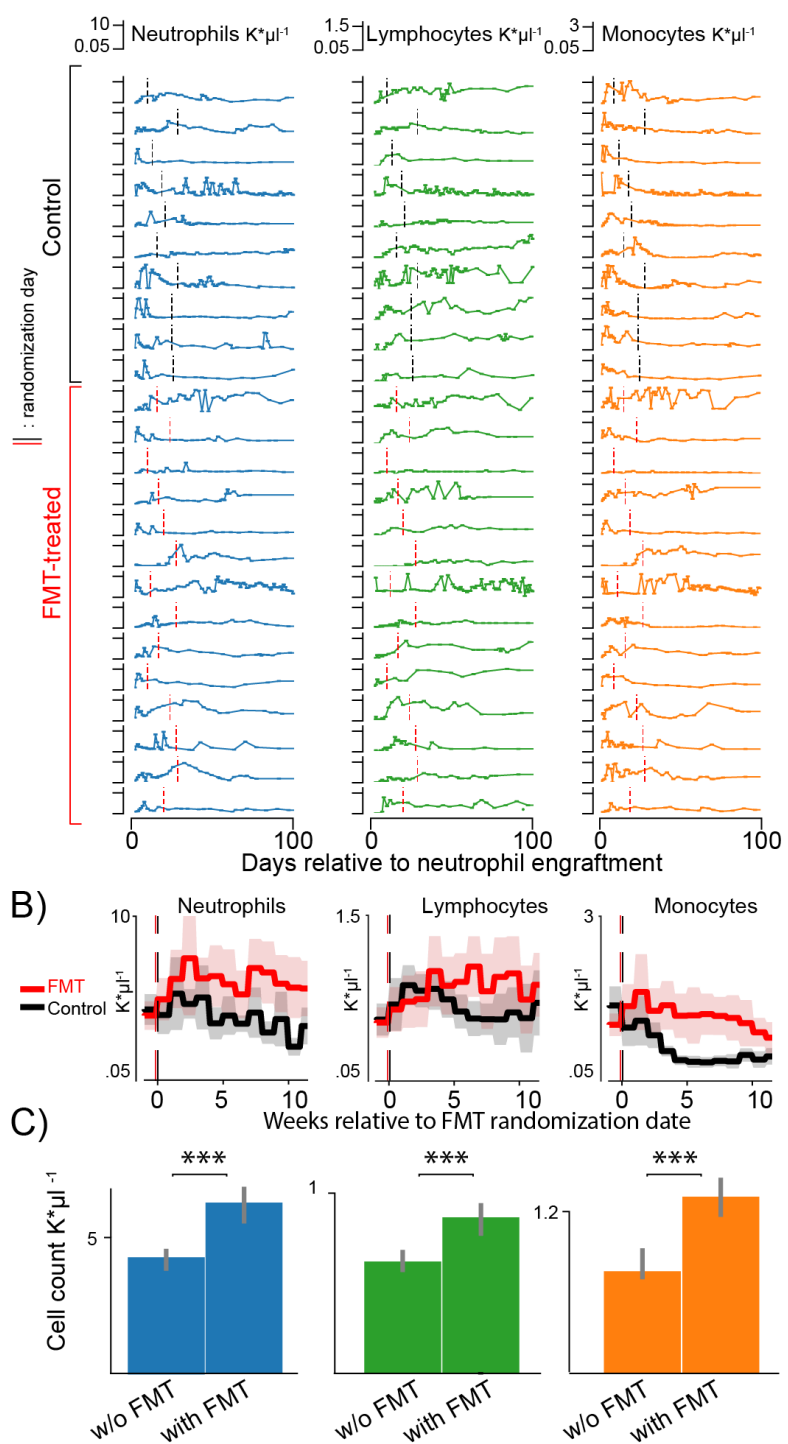

Neutrophils

Lymphocytes

Monocytes

Figure 2: Neutrophil, lymphocyte and monocyte counts increased in FMT-treated patients during the weeks following the treatment compared to control patients. A) Absolute counts of neutrophils (blue), lymphocytes (green) and monocytes (orange) in 24 patients enrolled in a randomized controlled trial to receive an autologous fecal microbiota transplant post-neutrophil engraftment (10 control: black vertical line, 14 FMT treated: red vertical line,). B) Weekly mean cell counts aligned to the date of randomization into FMT treatment arm (red) or control (black). Line: mean per week, shaded region: 95\%-CI. C) Results from a linear mixed effects model with random effects per patient and per day relative to neutrophil engraftment confirms that neutrophil, lymphocyte and monocyte counts are higher in patients receiving autoFMT after their treatment as compared to control patients after the randomization date; bars and confidence intervals for the averages of observed white blood cell counts without FMT and post-FMT $(* * *: p<0.001)$. 
The increased white blood cells in patients receiving auto-FMT could be due to the

119 reconstitution of a complex microbiota that we saw in these patients ${ }^{23}$ and the associated metabolic

120 capabilities $^{6,7,25}$, or it could be a systemic response to a severe therapy which introduced billions of

121 intestinal organisms at once via an enema (no enema was administered to control patients ${ }^{23}$ ).

122 Moreover, while the mixed-effects model accounted for patient-specific HCT treatments in this

123 randomized patient cohort, chance differences in extrinsic factors such as different

124 immunomodulatory drug exposures may have affected this result due to the small cohort size.

125 Nonetheless, observing that auto-FMT recipients had increased white blood cell counts supports the

126 notion that the microbiota can modulate the peripheral immune system. High counts of lymphocytes

127 during immune reconstitution has been associated with improved clinical outcomes ${ }^{27}$. Additionally, in

128 our HCT patients, a higher average level of white blood cells measured across a period of 100 days

129 after neutrophil engraftment (supplementary methods) confirms a positive association with 3-year

130 survival (hazard ratio: 0.91, p:0.04). Determining which taxa modulate immune dynamics could open

131 new ways to improve robust immune reconstitution, which is critical for clinical outcomes ${ }^{27-29}$.

132 To address this question, we next investigated the link between the gut microbiota and the

133 dynamics of white blood cell recovery in our large observational cohort of HCT patients. Homeostasis

134 of circulatory white blood cell counts is a complex, dynamic process: neutrophils, lymphocytes and

135 monocytes are formed and released into the blood de novo by differentiation of hematopoietic

136 progenitor cells from the bone marrow, and they can be mobilized from thymus and lymph nodes

137 (lymphocytes), spleen, liver and lungs (neutrophils); they can also migrate from the blood to other

138 tissues when needed ${ }^{30}$. These processes are dynamic sources and sinks of circulatory white blood

139 cells, and they can be modulated by drugs administered to patients receiving HCT. To identify factors

140 associated with these dynamic source- and sink-processes — including the microbiota—we developed

141 a two-stage approach analyzing the changes of white blood cell counts between two days (i.e. the

142 rates of cell count increases and decreases). Stage 1 served as a feature selection stage where we used

143 data of 1,096 patients (after filtering for qualifying samples and applying exclusion criteria, see

144 supplementary methods) without available microbiome information to identify associations between

145 clinical metadata, including immunomodulatory medications (methods), and changes of white blood 
146 cell counts from one day to the next (Figure 3A). Stage 2 was performed on data from an independent

147 cohort of 841 different patients at MSK from whom concurrent microbiome samples were available.

148 Stage 2, our main analysis, sought to reveal associations between the abundance of microbial taxa and

149 the daily changes in blood cell counts in context of immunomodulatory medications, additional

150 clinical metadata and the current state of the blood itself.

In stage 1 we calculated the changes in neutrophil, lymphocyte and monocyte counts during patients' recovery from $>20,000$ pairs of post-engraftment blood samples separated by a single day

153 (Figure 3B, S7-9, supplementary methods). Using a cross-validated feature selection approach, we

154 detected medications and HCT treatment parameters that were associated with different rates of

155 change in neutrophil, lymphocyte and monocyte counts, including, as expected, GCSF and the graft

156 stem cell sources (Figure S7-S9, Table S1).

157 During stage 2 we sought to identify associations between bacterial taxa of the gut microbiota

158 and the dynamics of immune cells in circulation. For this, we performed Bayesian inferences using

159 data from different sets of patients with available microbiome samples. Stage 1 had identified —as

160 expected - that stem cell graft sources are associated with immune reconstitution kinetics (e.g. cord

161 on average slower compared with $\mathrm{PBSC}^{31}$ ), and we therefore stratified our patients by graft source in

162 stage 2. The model of stage 2 now included microbial genera as predictors of observed changes in

163 white blood cell counts, in addition to the medications selected in stage 1, clinical features

164 (conditioning intensity, age, sex), and the current state of the blood in the form of counts of

165 neutrophils, lymphocytes, monocytes, eosinophils, and platelets. The data comprised 841 patients, but

166 approximately $60 \%$ of the stool samples paired with a daily change in white blood cell counts were

167 taken before neutrophil engraftment (Figure 3B, Table S1, supplementary methods), i.e. when blood

168 cells counts were zero. In total, we analyzed 2,615 post-engraftment observations of changes in

169 neutrophil counts during immune reconstitution (lymphocytes: 2,006, monocytes: 2,534) with paired

170 stool samples which provided a large sample of observed white blood cell dynamics (Figure 3B,

171 Table S3,S4). We first focused on the data from the largest (Figure 3C) cohort—patients who

172 received a PBSC graft - and withheld the other cohorts (bone marrow, BM; T-cell depleted graft

173 (ex-vivo) by CD34+selection, TCD; and cord) to use as independent validation cohorts. For this 
174 validation, we analyzed TCD, BM, and cord patients' data in the same way as PBSC patients' data

175 and compared the resulting posterior coefficient distributions (methods). We assigned coefficients

176 obtained from the PBSC cohort a validation score (v-score) between 0 and 3, representing the number

177 of times that the focal coefficient was validated in the other cohorts; but, conservatively, the score was

178 always set to zero if we observed counter-evidence among any of the other data sets, i.e. evidence that

179 coefficients had the opposite sign, ensuring only the most consistent associations were considered as

180 validated. Finally, we analyzed data from another patient cohort consisting of 493 bone marrow

181 transplantation recipients treated at Duke University including 9,603 blood samples and a total of 629

182 microbiota samples from 218 patients, albeit with lower sampling density, and we used the results

183 from this analysis for further validation.

184 Notably, as a verification of our approach, we detected associations between the

administration of immunomodulators and increased or decreased rates of immune cell count changes consistent with the known biological mechanism of these medications (Figure 3C,S10-S13). The strongest across all predictors is the well-known neutrophil-increasing effect of $\mathrm{GCSF}^{32}$; GCSF administration — used to accelerate recovery from chemotherapy-induced neutropenia ${ }^{32}$ — was associated with a $+140 \%$ increase in the rate of neutrophil changes from one day to the next $([+114 \%$, $+170 \%$ ], 95 percent probability density interval [HPDI95]). This finding was observed in all MSK validation data sets (v-score $=3$, Figure 3D), as well as among Duke University patients (Figure

S14,S15). We furthermore found a GCSF-associated increase of $+43 \%([+30 \%,+58 \%]$ HPDI $95, \mathrm{v}-$ score $=3)$ in monocyte rates, and, although smaller, in lymphocyte rates $(+16 \%,[+5 \%,+27 \%]$ HPDI95, $\mathrm{v}$-score $=3$ ). Both neutrophil and lymphocyte rates decreased following the exposure to antihistamine or immunosuppressive medications (cetirizine $-18 \%,[-35 \%,+5 \%]$ HPDI 95 , mycophenolate mofetil -8\% [-15\%,+1\%]HPDI95, respectively). Finally, less intensive chemotherapeutic conditioning regimens (non-ablative conditioning and reduced intensity) were associated with larger lymphocyte and monocyte count growth rates during immune reconstitution (Figure S10C) analysis detected associations between the current count of white blood cells and their rate of change: 
202 lymphocytes with the rates of monocytes, and a negative association between the counts of platelets

203 and lymphocytes and the rates of neutrophils (Figure 3E). Conversely, we found positive associations

204 between monocytes and the rates of each of the investigated white blood cell subsets. These

205 associations, derived from daily counts of white blood cells, could reflect a complex network

206 underlying the regulation of blood immune cell composition ${ }^{30}$. More importantly, the associations

207 quantified for medications and potential homeostatic feedbacks provided a benchmark against which

208 we could compare associations from gut microbial taxa.
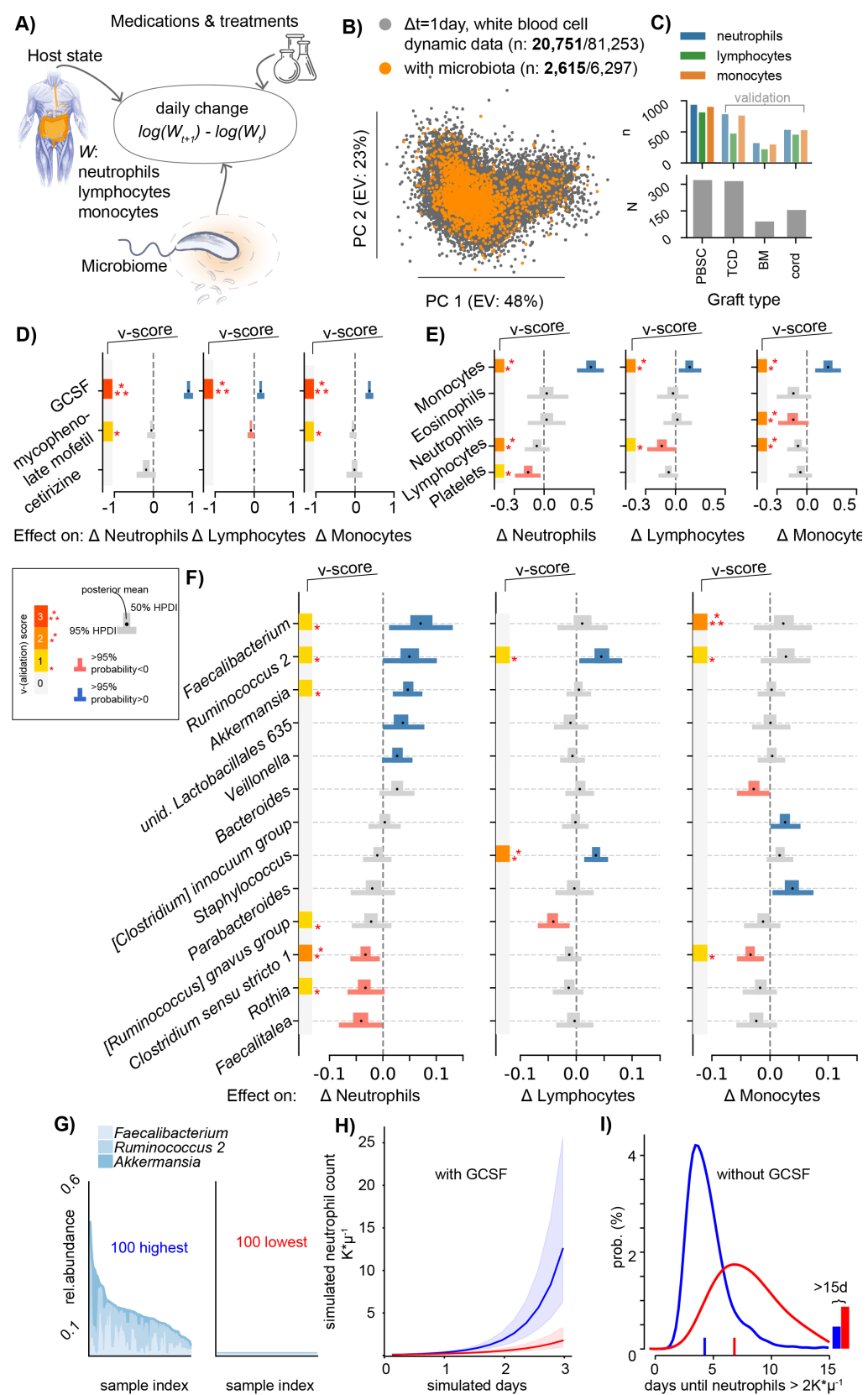
daily changes in circulatory white blood cell counts. A) Cartoon of the model: observed changes in white blood cell counts between two consecutive days are associated with the current state of the host in the form of blood cell counts in circulation, the administration of immunomodulatory medications, patient and clinical metadata, and the state of the microbiome. B) Visualization of the dynamic white blood cell data; scatter plot of the principal components (PC) of observed daily changes of neutrophils, lymphocytes and monocytes without (grey, see Figures S7-9) and with (orange) concurrent longitudinal microbiome data (bold: post engraftment sample counts). C) PBSC patients ( $\mathrm{N}=312$ ) provided the most blood samples with simultaneous microbiome data $(n=995)$ relative to TCD, BM and cord patients, who were used as validation data sets. D-F) PBSC patient data inference results; bars show the posterior parameter estimate distributions (thin: $95 \%$ highest posterior density intervals, HPDI95, thick: HPDI50) for the jointly inferred associations between treatments (D), white blood cells counts (E), and fecal microbiota genus log-relative abundances (F) with the observed daily changes in neutrophils, lymphocytes and monocytes. v-score: number of validation cohort confirming associations, always set to zero if invalidated in any of the TCD, BM, or cord cohorts (additional coefficients in Figure S10). G) 100 microbiota samples with highest (left) or lowest (right) relative abundances of Faecalibacterium, Ruminococcus 2 and Akkermansia. H) Simulation of the neutrophil population over time in presence of GCSF with microbiota compositions sampled either from those high (blue) or low (red) in Faecalibacterium, Ruminococcus 2 and Akkermansia relative abundance as shown in G); line: median of 1,000 simulations, shaded regions: quartile range of simulated neutrophil trajectories. I) In absence of GCSF, equivalent simulations to $\mathrm{H}$ ) predict that the time to reach neutrophil counts $>2 \mathrm{~K}^{*} \mu \mathrm{l}^{-1}$ for the first time after HCT when the microbiota is high (red) in Faecalibacterium, Ruminococcus 2 and Akkermansia compared with when these genera are low (blue) will decrease from 6.8 (95\%-confidence interval, CI: [6.5, 7]) days to 4.4, (CI: [4.3, 4.5]).

We identified microbial genera that consistently associated with increases or decreases in

white blood cell counts by first using data from the PBSC patients and then validating the associations in the other cohorts (Figure 3F). Higher abundances of Faecalibacterium $(+8 \%,[+1 \%$, $+14 \%]$ HPDI95 per $\left.\log _{10}\right)$, Ruminococcus $2(+5 \%,[0 \%,+10 \%]$ HPDI95) and Akkermansia $(+4 \%$,

$238[+1 \%,+7 \%]$ HPDI95) were associated with greater neutrophil increases, whereas increased Rothia (3\%, [-7\%, 0\%]HPDI95), and Clostridium sensu stricto $1(-3 \%,[-6 \%, 0 \%]$ HPDI95) relative

240 abundances associated with reduced neutrophil rates. These results were validated in univariate

241 analyses conducted in the Duke University cohort (Figure S14, S15). We also conducted the

242 inference using total genus abundances as predictors instead of relative abundances; this analysis confirmed Faecalibacterium as most strongly associated with neutrophil dynamics (Figure S16,

244 supplementary methods). Staphylococcus was positively associated with lymphocyte rates ( $+4 \%$,

$245[+1 \%,+6 \%]$ HPDI95) and, again, Ruminococcus 2 was also associated with faster lymphocyte

246 increases $(+5 \%,[+1 \%,+9 \%]$ HPDI95). Both Faecalibacterium as well as Ruminococcus 2 also

247 associated with increases in monocytes, and while this association was validated in other cohorts (v-

248 score 3 and 1, respectively), there was higher uncertainty of the association estimate (HPDI50>0).

249 Again, Clostridium sensu strictu 1 (-3\% [-5\%, -1\%]HPDI95) associated consistently with decreased

250 rates of monocytes. The associations we identified — and validated in other cohorts—between 
microbial taxa in the gut and daily changes in white blood cell counts support the idea that

252 hematopoiesis and mobilization respond to the composition of the gut microbiome, influencing systemic immunity ${ }^{33}$.

Most of the taxa that strongly associated with white blood cell dynamics were obligate anaerobes. Rothia, was a notable exception: this aerobic genus is typically found in the oral cavity ${ }^{34}$ but can become an opportunistic pathogen in immunosuppressed patients and is not known to provide metabolic functions to the host ${ }^{35}$. Some obligate anaerobes, on the other hand, produce short-chain fatty acids $\mathrm{s}^{36,37}$ and bacterial cell-wall molecules ${ }^{1,38,39}$ that modulate immune responses and granulopoiesis $^{6}$. Nutritional support from the intestinal microbiota improved hematopoietic reconstitution in a mouse mode $^{25}$. To identify a similar association in our patients, we estimated a microbiota potency by multiplying the $\log _{10}$-relative abundances of microbial genera in a sample with their corresponding posterior coefficients. We analyzed shotgun metagenomics sequences from 124 of the samples and observed that samples with positive microbiota potency were associated with enrichment in cholate degradation and vitamin-B1 synthesis related pathways, as well as butanoate formation (Figure S17). Our findings are in line with evolutionary theory ${ }^{40}$ that essential but broadly available microbial traits such as the production of B vitamins, secondary bile acid metabolism, and

267 fermentation to short-chain fatty acids ${ }^{41}$ could be co-opted by the host's immune system as part of the homeostatic interplay between immune system and a complex microbiota ${ }^{42,43}$. For example, release of energy from complex starch in the normal $\operatorname{diet}^{44}$. Reassuringly, the genera Faecalibacterium, Ruminococcus 2 and Akkermansia that we associated with faster rates of white

272 blood cells (Figure 3F) were among those best reconstituted by auto-FMT ${ }^{23}$, potentially explaining

273 why we found higher counts of neutrophils, monocytes and lymphocytes in patients who received the auto-FMT (Figure 2B,C).

The associations we reveal are interpretable as potential effectors on sources and sinks of white blood cell counts in circulation. Intestinal bacteria may affect white blood cell counts in circulation by influencing either their sources in the bone marrow or their cytokine profiles ${ }^{45}$ and

278 proliferation rates in the blood, their sinks in different organs, or both. The human immune system in 
turn can interact with the microbiota and modulate its composition, for example via

280 immunoglobulin A responses targeting specific bacteria as studied in mice $e^{43,46,47}$. To investigate a

281 reverse effect of the peripheral immune cell system onto bacterial populations, we employed an

282 analogous approach to the stage 1 analysis of white blood cell dynamics. Dynamics of white blood

283 cells can be estimated from changes in absolute cell counts, and to obtain the necessary measurements

284 in absolute bacterial abundances, we measured total bacterial 16S rRNA gene copies per gram of stool

285 for a subset of our samples (3,995 samples from 481 patients). Using absolute abundances of bacteria

286 as predictors in addition to medications, we jointly inferred the association network of dynamics

287 between the gut bacterial ecosystem and the peripheral immune system. All of our patients receive

288 antibiotics on some days during their treatment ${ }^{24}$ and their strong effects on microbiota dynamics

289 were the dominant effects that survived cross-validated regularized elastic net regression (Figure

290 S18). Relaxing the strength of the regularization (methods), however, revealed several bi-directional

291 relationships between immune cells in circulation and bacterial dynamics in the gut (Figure S19). Of

292 note, we detected a negative association of absolute [Ruminococcus] gnavus group abundance with

293 lymphocytes dynamics, confirming our main result based on relative bacterial abundances (Figure 3).

294 In the reverse direction, we saw a positive association of [Ruminococcus] gnavus group dynamics

295 with lymphocyte counts. This result agrees with findings that Ruminococcus gnavus thrives in and

296 promotes inflammatory conditions such as Crohn's disease and other inflammatory bowel diseases

297 (IBD) ${ }^{48}$; our analysis suggests it may drive high neutrophil to lymphocyte ratios that are broadly

298 characteristic for poor disease outcomes in $\operatorname{IBD}^{49,50}$ and beyond ${ }^{51,52}$.

299 Overall, our analysis identified that the microbiome is associated with immune cell dynamics

300 in addition to medications. The effects should be interpreted as net effects since they do not

301 distinguish, for example, how the microbiota impacts de novo hematopoiesis in isolation from its

302 impact on other sources and sinks. Unlike the plausible role of obligate anaerobe fermenters in

303 augmenting hematopoiesis via nutritional support $\mathrm{t}^{25}$, the positive association detected between

304 Staphylococcus and lymphocyte dynamics could instead result from reduced extravasation of T cells

305 from circulation into the gut epithelium ${ }^{53}$, especially since high abundances of Staphylococcus are 
306 associated with low gut microbiota diversity $(\mathrm{p}<0.001$, Figure S20), which indicates a depleted

307 microbiota.

308 Nevertheless, our approach allows us to leverage the chronology of events and assess

309 "mathematical causality" 54 . Of course, due to the observational nature of these data there are risks of

310 confounding that could explain some of the associations found, but the close temporal

311 correspondence ${ }^{54}$ between microbiota and blood cell dynamics, and the validation across cohorts

312 reduces the number of plausible confounders. Our results, therefore, quite naturally suggest candidate

313 microbial taxa to manipulate if we seek to steer complex hematopoietic dynamics and utilize the

314 microbiota as an immunomodulatory component of the human body. Intriguingly, members of

315 Faecalibacterium and Ruminococcus in one study ${ }^{10}$, and Akkermansia in another $^{9}$, were identified as

316 enriched in patients with better responses to anti-PD-1 immunotherapy, which suggested a

317 disagreement between the two studies ${ }^{55}$. Our results, however, revealed Faecalibacterium,

318 Ruminococcus 2, and Akkermansia as the most strongly associated taxa with increases in white blood

319 cell counts from one day to the next. Therefore, our results agree with the findings of both anti PD-1

320 therapy studies that these taxa are associated with immune modulation in humans. Our results also

321 allow us to compare the potency of manipulating these intestinal commensals to that of

322 immunomodulatory drugs. While these genera are common in the gut microbiota of healthy people ${ }^{17}$,

323 the relative abundance of each genus can drop below detection in our patients during the intestinal

324 damage related to $\mathrm{HCT}^{24}$. Therefore, realistic ranges of 3-5 orders of magnitude in bacterial log-

325 relative abundances (Figure 3G, S21) can yield effect sizes similar to that of homeostatic feedbacks

326 between white blood cells and several immunomodulatory medications (e.g. a change in

327 Ruminococcus 2 from below detection to $1 \%$ relative abundance associated with a $+67 \%$ and $+63 \%$

328 increases in neutrophil and lymphocyte rates, respectively). Therefore, while the effect sizes of

329 intestinal bacteria at first may appear smaller than those of immunomodulatory drugs, the herein

330 estimated homeostatic effects of gut bacteria may not be that small since their coefficients refer to

331 changes in exponential rates of white blood cells and accumulate each day. To better demonstrate how

332 this accumulation of effects would work, we conducted simulations of the inferred dynamic system of

333 white blood cells using our posterior coefficient distributions (methods). We simulated 1,000 time 
334 series for microbiota compositions either chosen from the 100 samples highest or lowest in

335 Faecalibacterium, Ruminococcus 2 and Akkermansia (Figure 3G), in presence (Figure 3H) or

336 absence (Figure 3I) of GCSF administration. Simulations predict that a microbiota enriched in these

337 genera could accelerate immune reconstitution, and reduce the time until neutrophils reach $>2 \mathrm{~K}^{*} \mu \mathrm{l}^{-1}$

338 in absence of GCSF by 2.4 days, from predicted 6.8 (CI: [6.5, 7]) to 4.4 days (CI: [4.3,4.5]) days. Gut

339 bacteria, in concert and over time, could therefore have significant impact on systemic immunity even

340 in individuals with less severely injured microbiomes.

341 In sum, our work links the gut microbiota to the dynamics of the human immune system via

342 peripheral white blood cell populations. Our analysis uses white blood cells counted directly from

343 patients, which are coarse-grained clinical analyses conducted at large scale but lack details such as

344 lymphocyte and other immune cell subsets. Nonetheless, because it is in humans, this study fills an

345 important gap at a critical time for microbiome research when the clinical relevance of animal models

346 of microbiome-immune interaction has been questioned ${ }^{21}$. By studying a large number of patients

347 over time, we were able to infer and quantify for the first time the association of microbiota

348 components on systemic immune cell dynamics, and our results help to consolidate previous

349 findings ${ }^{10,9}$ that seemed in conflict with each other ${ }^{55}$. Our study demonstrates that the composition of

350 the microbiota does indeed modulate systemic immune cell dynamics, a link that could be used in the

351 future to improve immunotherapy and help identify microbiota treatments for inflammatory

352 diseases ${ }^{9,10,56-60}$. 
1. Mazmanian, S. K., Liu, C. H., Tzianabos, A. O. \& Kasper, D. L. An immunomodulatory molecule of symbiotic bacteria directs maturation of the host immune system. Cell 122, 107-118 (2005).

2. Gomez de Agüero, M. et al. The maternal microbiota drives early postnatal innate immune development. Science 351, 1296-1302 (2016).

3. Sonnenberg, G. F. \& Artis, D. Novel connections and precision approaches. Nat. Rev. Immunol. 19, 75-76 (2019).

4. Geva-Zatorsky, N. et al. Mining the human gut microbiota for immunomodulatory organisms. Cell 168, 928-943.e11 (2017).

5. Tan, T. G. et al. Identifying species of symbiont bacteria from the human gut that, alone, can induce intestinal Th17 cells in mice. Proc. Natl. Acad. Sci. USA 113, E8141-E8150 (2016).

6. Balmer, M. L. et al. Microbiota-derived compounds drive steady-state granulopoiesis via MyD88/TICAM signaling. J. Immunol. 193, 5273-5283 (2014).

7. Deshmukh, H. S. et al. The microbiota regulates neutrophil homeostasis and host resistance to Escherichia coli K1 sepsis in neonatal mice. Nat. Med. 20, 524-530 (2014).

8. Tanoue, T. et al. A defined commensal consortium elicits CD8 T cells and anti-cancer immunity. Nature 565, 600-605 (2019).

9. Routy, B. et al. Gut microbiome influences efficacy of PD-1-based immunotherapy against epithelial tumors. Science 359, 91-97 (2018).

10. Gopalakrishnan, V. et al. Gut microbiome modulates response to anti-PD-1 immunotherapy in melanoma patients. Science 359, 97-103 (2018).

11. Vétizou, M. et al. Anticancer immunotherapy by CTLA-4 blockade relies on the gut microbiota. Science 350, 1079-1084 (2015).

12. Matson, V. et al. The commensal microbiome is associated with anti-PD-1 efficacy in metastatic melanoma patients. Science 359, 104-108 (2018).

13. Atarashi, K. et al. ATP drives lamina propria $\mathrm{T}(\mathrm{H}) 17$ cell differentiation. Nature $\mathbf{4 5 5}$, 808-812 (2008).

14. Ivanov, I. I. et al. Specific microbiota direct the differentiation of IL-17-producing Thelper cells in the mucosa of the small intestine. Cell Host Microbe 4, 337-349 (2008).

15. Cahenzli, J., Köller, Y., Wyss, M., Geuking, M. B. \& McCoy, K. D. Intestinal microbial diversity during early-life colonization shapes long-term IgE levels. Cell Host Microbe 14, 559-570 (2013).

16. Lloyd-Price, J. et al. Multi-omics of the gut microbial ecosystem in inflammatory bowel diseases. Nature 569, 655-662 (2019).

17. Integrative HMP (iHMP) Research Network Consortium. The integrative human microbiome project. Nature 569, 641-648 (2019).

18. Olin, A. et al. Stereotypic immune system development in newborn children. Cell 174, 1277-1292.e14 (2018).

19. Brandi, G. \& Frega, G. Microbiota: Overview and Implication in Immunotherapy-Based Cancer Treatments. Int. J. Mol. Sci. 20, (2019).

20. Xin Yu, J., Hubbard-Lucey, V. M. \& Tang, J. The global pipeline of cell therapies for cancer. Nat. Rev. Drug Discov. 18, 821-822 (2019).

21. Walter, J., Armet, A. M., Finlay, B. B. \& Shanahan, F. Establishing or Exaggerating 
Causality for the Gut Microbiome: Lessons from Human Microbiota-Associated Rodents. Cell 180, 221-232 (2020).

22. Taur, Y. et al. Intestinal domination and the risk of bacteremia in patients undergoing allogeneic hematopoietic stem cell transplantation. Clin. Infect. Dis. 55, 905-914 (2012).

23. Taur, Y. et al. Reconstitution of the gut microbiota of antibiotic-treated patients by autologous fecal microbiota transplant. Sci. Transl. Med. 10, (2018).

24. Morjaria, S. et al. Antibiotic-Induced Shifts in Fecal Microbiota Density and Composition during Hematopoietic Stem Cell Transplantation. Infect. Immun. 87, (2019).

25. Staffas, A. et al. Nutritional Support from the Intestinal Microbiota Improves Hematopoietic Reconstitution after Bone Marrow Transplantation in Mice. Cell Host Microbe 23, 447-457.e4 (2018).

26. Taur, Y. et al. The effects of intestinal tract bacterial diversity on mortality following allogeneic hematopoietic stem cell transplantation. Blood 124, 1174-1182 (2014).

27. Savani, B. N. et al. Absolute lymphocyte count on day 30 is a surrogate for robust hematopoietic recovery and strongly predicts outcome after $\mathrm{T}$ cell-depleted allogeneic stem cell transplantation. Biol. Blood Marrow Transplant. 13, 1216-1223 (2007).

28. Mehta, R. S. \& Rezvani, K. Immune reconstitution post allogeneic transplant and the impact of immune recovery on the risk of infection. Virulence 7, 901-916 (2016).

29. Kim, H. T. et al. Absolute lymphocyte count recovery after allogeneic hematopoietic stem cell transplantation predicts clinical outcome. Biol. Blood Marrow Transplant. 21, 873-880 (2015).

30. Scheiermann, C., Frenette, P. S. \& Hidalgo, A. Regulation of leucocyte homeostasis in the circulation. Cardiovasc. Res. 107, 340-351 (2015).

31. Thompson, P. A. et al. Umbilical cord blood graft engineering: challenges and opportunities. Bone Marrow Transplant. 50 Suppl 2, S55-62 (2015).

32. Gabrilove, J. L. et al. Effect of granulocyte colony-stimulating factor on neutropenia and associated morbidity due to chemotherapy for transitional-cell carcinoma of the urothelium. N. Engl. J. Med. 318, 1414-1422 (1988).

33. Belkaid, Y. \& Hand, T. W. Role of the microbiota in immunity and inflammation. Cell 157, 121-141 (2014).

34. Hall, M. W. et al. Inter-personal diversity and temporal dynamics of dental, tongue, and salivary microbiota in the healthy oral cavity. npj Biofilms and Microbiomes 3, 2 (2017).

35. Ramanan, P., Barreto, J. N., Osmon, D. R. \& Tosh, P. K. Rothia bacteremia: a 10-year experience at Mayo Clinic, Rochester, Minnesota. J. Clin. Microbiol. 52, 3184-3189 (2014).

36. Ulven, T. Short-chain free fatty acid receptors FFA2/GPR43 and FFA3/GPR41 as new potential therapeutic targets. Front. Endocrinol. (Lausanne) 3, 111 (2012).

37. Smith, P. M. et al. The microbial metabolites, short-chain fatty acids, regulate colonic Treg cell homeostasis. Science 341, 569-573 (2013).

38. Hergott, C. B. et al. Peptidoglycan from the gut microbiota governs the lifespan of circulating phagocytes at homeostasis. Blood 127, 2460-2471 (2016).

39. Ladinsky, M. S. et al. Endocytosis of commensal antigens by intestinal epithelial cells regulates mucosal T cell homeostasis. Science 363, (2019).

40. Foster, K. R., Schluter, J., Coyte, K. Z. \& Rakoff-Nahoum, S. The evolution of the host microbiome as an ecosystem on a leash. Nature 548, 43-51 (2017).

41. Yoshii, K., Hosomi, K., Sawane, K. \& Kunisawa, J. Metabolism of dietary and microbial vitamin B family in the regulation of host immunity. Front. Nutr. 6, 48 (2019).

42. Zaza, G. et al. Impact of maintenance immunosuppressive therapy on the fecal microbiome of renal transplant recipients: Comparison between an everolimus- and a 
standard tacrolimus-based regimen. PLoS One 12, e0178228 (2017).

43. McLoughlin, K., Schluter, J., Rakoff-Nahoum, S., Smith, A. L. \& Foster, K. R. Host selection of microbiota via differential adhesion. Cell Host Microbe 19, 550-559 (2016).

44. Ze, X., Duncan, S. H., Louis, P. \& Flint, H. J. Ruminococcus bromii is a keystone species for the degradation of resistant starch in the human colon. ISME J. 6, 1535-1543 (2012).

45. Schirmer, M. et al. Linking the human gut microbiome to inflammatory cytokine production capacity. Cell 167, 1125-1136.e8 (2016).

46. Hooper, L. V., Littman, D. R. \& Macpherson, A. J. Interactions between the microbiota and the immune system. Science 336, 1268-1273 (2012).

47. Palm, N. W. et al. Immunoglobulin A coating identifies colitogenic bacteria in inflammatory bowel disease. Cell 158, 1000-1010 (2014).

48. Henke, M. T. et al. Ruminococcus gnavus, a member of the human gut microbiome associated with Crohn's disease, produces an inflammatory polysaccharide. Proc. Natl. Acad. Sci. USA 116, 12672-12677 (2019).

49. Okba, A. M. et al. Neutrophil/lymphocyte ratio and lymphocyte/monocyte ratio in ulcerative colitis as non-invasive biomarkers of disease activity and severity. Auto Immun. Highlights 10, 4 (2019).

50. Celikbilek, M. et al. Neutrophil-lymphocyte ratio as a predictor of disease severity in ulcerative colitis. J Clin Lab Anal 27, 72-76 (2013).

51. Gao, Y. et al. Neutrophil/lymphocyte ratio is a more sensitive systemic inflammatory response biomarker than platelet/lymphocyte ratio in the prognosis evaluation of unresectable pancreatic cancer. Oncotarget 8, 88835-88844 (2017).

52. Choi, S.-J. et al. High neutrophil-to-lymphocyte ratio predicts short survival duration in amyotrophic lateral sclerosis. Sci. Rep. 10, 428 (2020).

53. Fu, Y.-Y. et al. T Cell Recruitment to the Intestinal Stem Cell Compartment Drives Immune-Mediated Intestinal Damage after Allogeneic Transplantation. Immunity 51, 90-103.e3 (2019).

54. Gerber, G. K. The dynamic microbiome. FEBS Lett. 588, 4131-4139 (2014).

55. Jobin, C. Precision medicine using microbiota. Science 359, 32-34 (2018).

56. Zitvogel, L., Ma, Y., Raoult, D., Kroemer, G. \& Gajewski, T. F. The microbiome in cancer immunotherapy: Diagnostic tools and therapeutic strategies. Science 359, 13661370 (2018).

57. Hsiao, E. Y. et al. Microbiota modulate behavioral and physiological abnormalities associated with neurodevelopmental disorders. Cell 155, 1451-1463 (2013).

58. Lakritz, J. R. et al. Gut bacteria require neutrophils to promote mammary tumorigenesis. Oncotarget 6, 9387-9396 (2015).

59. Lakritz, J. R. et al. Beneficial bacteria stimulate host immune cells to counteract dietary and genetic predisposition to mammary cancer in mice. Int. J. Cancer 135, 529-540 (2014).

60. Chen, H. et al. A forward chemical genetic screen reveals gut microbiota metabolites that modulate host physiology. Cell 177, 1217-1231.e18 (2019).

61. Caporaso, J. G. et al. Ultra-high-throughput microbial community analysis on the Illumina HiSeq and MiSeq platforms. ISME J. 6, 1621-1624 (2012).

62. Callahan, B. J. et al. DADA2: High-resolution sample inference from Illumina amplicon data. Nat. Methods 13, 581-583 (2016).

63. Murali, A., Bhargava, A. \& Wright, E. S. IDTAXA: a novel approach for accurate taxonomic classification of microbiome sequences. Microbiome 6, 140 (2018).

64. Quast, C. et al. The SILVA ribosomal RNA gene database project: improved data processing and web-based tools. Nucleic Acids Res. 41, D590-6 (2013). 
65. Pinheiro, J. C., Bates, D. M., DebRoy, S. S. \& Sarkar, D. Nlme: Linear and Nonlinear Mixed Effects Models. (2013).

66. Tibshirani, R. Regression shrinkage and selection via the lasso. Journal of the Royal Statistical Society: Series B (Methodological) 58, 267-288 (1996).

67. Pedregosa, F. et al. Scikit-learn: Machine Learning in Python. Journal of Machine Learning Research (2011).

68. Salvatier, J., Wiecki, T. V. \& Fonnesbeck, C. Probabilistic programming in Python using PyMC3. PeerJ Computer Science 2, e55 (2016).

69. Hoffman, M. D. \& Gelman, A. The No-U-Turn Sampler: Adaptively Setting Path Lengths in Hamiltonian Monte Carlo. Journal of Machine Learning Research (2014).

70. Franzosa, E. A. et al. Species-level functional profiling of metagenomes and metatranscriptomes. Nat. Methods 15, 962-968 (2018).

71. Vehtari, A., Gelman, A., Simpson, D., Carpenter, B. \& Bürkner, P.-C. Ranknormalization, folding, and localization: An improved \$ widehat $\{\mathrm{R}\} \$$ for assessing convergence of MCMC. arXiv (2019). 
Appended and available online.

\section{Acknowledgments and Conflicts of Interest}

523 We thank Marc Lipsitch, Sandra B. Andersen, Kevin R. Foster, Jonathan Kevin Sia, Eric G. Pamer,

524 Kat Coyte, Sibylle Mitschka and the members of the Xavier lab for helpful discussion and comments 525 on the manuscript. This work was supported by the National Institutes of Health (NIH) grant U01 526 AI124275 to JBX and grant R01 AI137269 to JBX, by the MSKCC Cancer Center Core Grant P30 527 CA008748, the Parker Institute for Cancer Immunotherapy at Memorial Sloan Kettering Cancer

528 Center, the Sawiris Foundation, the Society of Memorial Sloan Kettering Cancer Center, MSKCC

529 Cancer Systems Immunology Pilot Grant and Empire Clinical Research Investigator Program. MS

530 received funding from the Burroughs Wellcome Fund Postdoctoral Enrichment Program, the Damon

531 Runyon Physician-Scientist Award, and the Robert Wood Johnson Foundation. MRMvdB and JUP

532 received financial support from Seres Therapeutics. TMH is investigator in the Pathogenesis of

533 Infectious Diseases from the Burroughs Wellcome Fund, and funded via an award from Geoffrey

534 Beene Foundation, and NIH RO1 AI093808. M-AP has received honoraria from AbbVie, Bellicum,

535 Bristol-Myers Squibb, Incyte, Merck, Novartis, Nektar Therapeutics, and Takeda; has received

536 research support for clinical trials from Incyte, Kite (Gilead) and Miltenyi Biotec; and serves on data

537 and safety monitoring boards for Servier and Medigene and scientific advisory boards for MolMed

538 and NexImmune. The funders had no role in study design, data collection and analysis, decision to

539 publish, or preparation of the manuscript.

\section{Author Contributions}

542 J.S. and J.B.X. wrote the manuscript. J.S. and J.B.X. designed the analyses with expert help from 543 R.N.. J.U.P. and Y.T. contributed to the clinical data preparation, B.P.T. provided the 16S data 544 processing pipelines, A.D. provided the shotgun processing pipelines. All authors contributed to the 545 writing and interpretation of the results. 
547 Methods

\section{Complete blood count collection and characterization}

549 Absolute white blood cells count data were obtained from routine complete blood counts ordered by

550 clinicians during normal clinical practice, used to obtain informative diagnostic and monitoring

551 information. Blood samples received in the clinical hematology laboratory were analyzed using

552 Sysmex XN automated hematology analyzers (Sysmex, Lincolnshire, IL) and, when needed based on

553 specific flags and parameters as per MSKCC standard operating procedures, were validated manually

554 using the Sysmex DI-60 Slide Processing System or CellaVision DM9600 Automated Digital

555 Morphology System (Sysmex, Lincolnshire, IL).

556

557

16S rRNA gene amplification and multiparallel sequencing

558 For each sample, duplicate 50- $\mu 1$ PCRs were performed, each containing $50 \mathrm{ng}$ of purified DNA, 0.2

$559 \mathrm{mM}$ deoxynucleotide triphosphates, $1.5 \mathrm{mM} \mathrm{MgCl} 2,2.5 \mathrm{U}$ Platinum Taq DNA polymerase, $2.5 \mu \mathrm{l}$ of

$56010 \times$ PCR buffer, and $0.5 \mu \mathrm{M}$ of each primer designed to amplify the V4-V5: 563F (5'-nnnnnnnn-

561 NNNNNNNNNNNN-AYTGGGYDTAAAGNG-3') and 926R (5'-nnnnnnnn-NNNNNNNNNNNN-

562 CCGTCAATTYHTTTRAGT-3'). A unique 12-base Golay barcode (Ns) precedes the primers for

563 sample identification ${ }^{61}$, and one to eight additional nucleotides were placed in front of the barcode to

564 offset the sequencing of the primers. Cycling conditions were $94^{\circ} \mathrm{C}$ for $3 \mathrm{~min}$, followed by 27 cycles

565 of $94^{\circ} \mathrm{C}$ for $50 \mathrm{~s}, 51^{\circ} \mathrm{C}$ for $30 \mathrm{~s}$, and $72^{\circ} \mathrm{C}$ for $1 \mathrm{~min}$. For the final elongation step, $72^{\circ} \mathrm{C}$ for 5 min was

566 used. Replicate PCRs were pooled, and amplicons were purified using the QIAquick PCR Purification

567 Kit (Qiagen). PCR products were quantified and pooled at equimolar amounts before Illumina

568 barcodes and adaptors were ligated, using the Illumina TruSeq Sample Preparation protocol. The

569 completed library was sequenced on an Illumina MiSeq platform following the Illumina

570 recommended procedures with a paired-end $250 \times 250 \mathrm{bp}$ kit

571

572 Sequence analysis 
573 The 16S (V4-V5) paired-end reads were merged and demultiplexed. Amplicon sequence variants

574 (ASVs) were identified using the Divisive Amplicon Denoising Algorithm (DADA2) pipeline

575 including filtering and trimming of the reads ${ }^{62}$. Reads were trimmed to the first $180 \mathrm{bp}$ or the first

576 point with a quality score $\mathrm{Q}<2$. Reads were removed if they contained ambiguous nucleotides $(\mathrm{N})$ or

577 if two or more errors were expected based on the quality of the trimmed read. We assigned taxonomy

578 to ASVs using a 8-mer based classifier trained by IDTaxa ${ }^{63}$ using the SILVA database ${ }^{64}$. We

579 determined the copy number of 16S rRNA genes per gram of stool for 4,158 of our samples as

580 reported previously ${ }^{24}$, by quantitative PCR on total DNA extracted from fecal samples.

581

582 Quantification of total microbiota density per gram of stool and estimation of total genus

583 abundances.

584 Quantitative PCR (qPCR) was performed on DNA extracted from the 1g wet weight of a stool sample 585 using DyNAmo SYBR Green qPCR kit (Finnzymes) and $0.2 \mu \mathrm{M}$ of the universal bacterial primer 8F

586 (5'-AGAGTTTGATCCTGGCTCAG) and the broad-range bacterial primer 338R

587 (5'-TGCTGCCTCCCGTAGGAGT-3'). Standard curves were prepared by serial dilution of the PCR

588 blunt vector (Invitrogen) containing 1 copy of the 16 s rRNA gene. Cycling conditions were $95^{\circ} \mathrm{C}$ for

58910 minutes followed by 40 cycles of $95^{\circ} \mathrm{C}$ for 30 seconds, $52^{\circ} \mathrm{C}$ for 30 seconds, and $72{ }^{\circ} \mathrm{C}$ for 1

590 minute. We used the measurements of total 16S rRNA gene counts per gram of stool to multiply the

591 relative abundances of taxa obtained from $16 \mathrm{~S}$ amplicon sequencing to obtain the estimate of their

592 total abundance per gram of stool (supplementary methods). Importantly, this does not account for

593 16S copy number variation between taxa, but the observed dynamic ranges in total abundances of taxa

594 in our data set span up to 9 orders of magnitude, exceeding the potential inaccuracies due to copy

595 number variation.

596

597 Diversity calculations 
598 Microbiome alpha-diversity was measured by the inverse Simpson (IS) index of a sample. It was

599 calculated by $\mathrm{IS}_{\mathrm{i}}=\frac{1}{\sum_{j=1}^{N} p_{i j}^{2}}$, where $p$ is the relative abundance of the $j$ th ASV out of $N$ total ASVs in 600 sample $i$.

601

\section{Linear mixed-effects model of white blood cell counts}

603 To study the effect of auto-FMT on white blood cells, we investigated the white blood cell counts of

60424 enrolled patients of this trial from the day of neutrophil engraftment until 100 days after. FMT

605 occurred on different days relative to neutrophil engraftment. Thus, we performed an analogous

606 analysis to that conducted in the original publication that demonstrated how FMT re-established a

607 diverse microbiome in the post-FMT period ${ }^{23}$. To answer if white blood cell counts differed post-

608 FMT, we used a linear mixed effects model of white blood cell counts, $y$, modeled as a function of the

609 FMT treatment as well as patient and timepoint specific random effects. We included random

610 intercept terms for each day $i$ and each patient $j$, and a fixed effects term for the post-FMT period with

611 associated coefficient "armpost", using the indicator variable "FMT", that is 1 when a patient was

612 from the FMT treated arm of the trial and day was greater than or equal to the day of the FMT

613 procedure. We conducted independent analyses for neutrophil, lymphocyte and monocyte counts.

614 This resulted in the following model of a cell count, $y$, for patient $j$ on day $i$ :

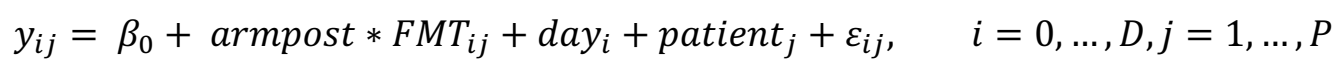

616 with prior distributions $\operatorname{day}_{i} \sim \mathcal{N}\left(0, \sigma_{\text {day }}^{2}\right)$, and patient $_{j} \sim \mathcal{N}\left(0, \sigma_{\text {patient }}^{2}\right)$, independent error

$617 \varepsilon_{i j} \sim \mathcal{N}\left(0, \sigma^{2}\right)$ and fixed intercept $\beta_{0}$, for the $\mathrm{D}$ days post neutrophils engraftment and $\mathrm{P}$ patients,

$618(\mathrm{D}=100, \mathrm{P}=24)$. For convenience of those interested in reanalyzing our data, the part of our data

619 concerning the auto-FMT analysis is available in tidy format (supplementary data 11), and the

620 analysis code conducted in the R programming language is available as an exported notebook

621 (fmt_effect_on_wbc.pdf) on github: https://github.com/jsevo/wbcdynamics_microbiome/. ${ }^{65} \mathrm{We}$

622 conducted an additional analysis with "day" as a continuous predictor which did not change our

623 conclusions (supplementary methods). 


\section{Dynamic systems analyses}

626 We analyzed factors associated with the observed changes of absolute counts of neutrophils,

627 lymphocytes and monocytes between two days. In the following we describe how chronology of

628 events and biological samples were encoded, and the models used to infer a role of medications,

629 clinical parameters and the microbiome on dynamics of white blood cells.

630 To reveal factors that associate with day-to-day changes in white blood cell counts, we started

631 from a first-order differential equation of white blood cell (W) dynamics:

$$
\frac{\mathrm{d}(\mathrm{W})}{\mathrm{d} t}=W\left(g r+\sum_{j=1}^{P} \beta_{j} X_{j}\right)
$$

633 Where $g r$ represents the intercept, i.e. the base line rate of change during immune reconstitution, and

$634 \beta_{j}$ are the to-be-estimated coefficients of the $P$ predictors $X_{j}, j \in P$, of the white blood cell dynamics.

635 This equation was then linearized to

$$
\frac{\mathrm{d} \ln (\mathrm{W})}{\mathrm{d} t}=g r+\sum_{j=1}^{P} \beta_{j} X_{j}
$$

637 And we parameterized the corresponding discrete difference equation:

$$
\frac{\Delta \ln (\mathrm{W})}{\Delta t}=g r+\sum_{j=1}^{P} \beta_{j} X_{j}
$$

where $\Delta \ln (\mathrm{W})$ is the log-difference between single days of neutrophils, lymphocytes or monocytes

640 counts, and $\Delta \mathrm{t}=1$ for all intervals. Predictors include the counts of neutrophils, lymphocytes,

641 monocytes, eosinophils and platelets during an interval (homeostatic feedbacks), immunomodulatory

642 medication and clinical observations such as a blood stream infection and the onset of graft versus

643 host disease, HCT parameters such as graft types and conditioning regimens, and, additionally, the

644 microbiota composition in "stage 2" of our analysis (supplementary methods for data exclusion and

645 additional details on interval definitions). Importantly, by parameterizing a dynamic equation and

646 analyzing rates of change, our coefficient estimates have an immediate causal interpretation within

647 our modeling framework (i.e. a $\beta_{j}>0$ implies that higher levels of the corresponding $X_{j}$ increases the 
respective white blood cell type, $W$ ). To differentiate such results from other associations, analyses of this type have been termed "mathematical causality"

650

Stage 1 analysis: Feature selection. Identifying medications and clinical observations associated with

Stage 1 uses data of patients without any available microbiome samples and the following model of white blood cell changes, y:

$$
y=g r+\sum_{j=1}^{p} \beta_{j} X_{j}
$$

with intercept, $g r$. The predictors, $X$, include dummy variables for the HCT graft type, patients' age on

the date of HCT, sex, 13 most frequently observed positive blood cultures with remaining other blood stream infections grouped into a separate category "other infections", an indicator for the onset of graft versus host disease, administrations of 55 different, most common immunomodulatory medications and platelet transfusion events, and HCT conditioning intensity regimens as well as the

661 log-transformed geometric mean counts of neutrophils, lymphocytes, monocytes, eosinophils and platelets during the respective interval. We used elastic net regression ${ }^{66}$ for feature selection using the sklearn package for the Python programming language ${ }^{67}$. For elastic net regression with $50 \%$ L1penalty, predictors were scaled between zero and 1 , and we used 10 -fold cross validation (i.e. leaving out $10 \%$ of patients at each cross-validation step) to choose the regularization strength, $\lambda$, solving for

$$
\operatorname{argmin}_{g r, \beta}\left\{\frac{1}{2 N} \sum_{i=1}^{N}\left(y_{i}-g r-\sum_{j=1}^{p} x_{i j} \beta_{j}\right)^{2}+\frac{1}{2} \lambda \sum_{j=1}^{p}\left|\beta_{j}\right|+\frac{1}{2} \lambda \sum_{j=1}^{p} \beta_{j}^{2}\right\}
$$

Expanded analysis on patients with microbiome data - stage 2

671 To identify associations between microbiota and white blood cell dynamics, we conducted an

672 analogous, Bayesian regression using the package PyMC3 for the Python programming language ${ }^{68}$.

673 Stage 1 identified important difference between transplant types, and we therefore stratified our data 
675 we applied "no U-turn" sampling ${ }^{69}$ to produce 10,000 posterior samples from 5 independent MCMC

676 chains that parameterized the model:

$$
y \sim \mathcal{N}\left(\mu, \sigma^{2}\right)
$$

with uninformative prior distributions

$$
g r \sim \mathcal{N}(\text { mean }=0, \text { standard deviation }=100)
$$

$$
\beta_{j} \sim \mathcal{N}(\text { mean }=0, \text { standard deviation }=100)
$$

$$
\sigma \sim \text { HalfCauchy }(\text { beta }=2)
$$

683

where $y$ is the observed daily change of a focal white blood cell type as in stage 1 with normal

distributed mean, $\mu$, and $\sigma$, the model uncertainty with a thick-tailed half Cauchy prior (importantly, our posterior estimates do not depend on this choice as we obtain the same results with an inverse Gamma prior, figure S19). $\mu$ was a function of the baseline growth rate, $g r$, and predictors, $\widehat{P}$ : medications with non-zero coefficients in stage 1 , the white blood cell counts, patient age and sex, and HCT conditioning intensities; additionally, $\widehat{P}$ now included the log-abundances of microbial genera as measured by $16 \mathrm{~S}$ sequencing from DNA in the stool collected on the second day of a daily interval (see supplementary methods for details). We considered taxa that were among the 100 most abundant, or had reached maximum relative abundances of at least $10 \%$, and selected those who were non-zero in more than $75 \%$ of our samples. White blood cell counts and microbiota data present during a daily interval were log-transformed, and zeros were filled with half of the minimum observed non zero counts (i.e. $0.5 \mathrm{e} 3$ and 2e-6, respectively). We focused on the largest cohort (PBSC) and used the independent inference results from TCD, BM, and cord cohorts for validation.

\section{Validation score}

698 Coefficients learnt from the PBSC patient cohort were assigned a "validation score" based on the results obtained from the other three MSK patient cohorts. Our requirements for validation were conservative; we required evidence from our validation data sets as well as absence of counter 
evidence. For regression results from each of the validation graft type cohorts, i.e. TCD, BM, and cord, we checked if a coefficient had more than $75 \%$ probability $(50 \% \mathrm{HPDI})$ to have the same sign as the mean of the PBSC coefficient posterior for a given predictor. If so, this was considered evidence of validation, and we summed the evidence over the three validation sets (i.e. maximum score of 3,1 from each of TCD, BM, and cord cohorts). Conversely, if we found more than $75 \%$ probability among any of the validation data sets that a given predictor had the opposite sign as the posterior mean calculated from PBSC data, this was considered counter evidence and the validation score was always set to zero.

Analysis of white blood cell dynamics with absolute bacterial abundances as predictors instead of

\section{1 relative abundances}

712 We conducted an ordinary least squares regression using the statsmodels package in the Python programming language of the same model as in the main Bayesian analysis using total bacterial

714 abundances as predictors. This was only possible on a subset of 389 neutrophil, 331 lymphocyte and

715376 monocyte rate observations from PBSC patients.

\section{Forwards simulation of predicted immune system reconstitution kinetics}

718 To assess the impact of the estimated microbiota coefficients on immune system dynamics, we conducted 1,000 simulations of the system of 3 differential equations describing the dynamics of neutrophils, lymphocytes and monocytes. We ran 1,000 simulations four times: in presence and absence of GCSF, each with microbiota compositions enriched or depleted in Faecalibacterium,

Ruminococcus 2 and Akkermansia. To identify these compositions, we ranked the observed microbiota compositions by these taxa, and chose randomly either from the top or bottom 100 . The coefficients for white blood cell interactions, interactions with the microbiota and the effect of GCSF were sampled from our posterior coefficient distributions. Using these coefficients sampled at the start of the simulation, and using 50 cells* $^{*} \mathrm{l}^{-1}$ of neutrophils, lymphocytes and monocytes as initial values, we simulated these differential equations forwards in time using the odeint function of the scipy package for the Python programming language. 
Validation on data from Duke University

731 We analyzed 9,603 blood samples with 25,581 associated administrations of immunomodulatory medications, and 741 microbiota samples from Duke as an orthogonal data set to validate our relevant post neutrophil engraftment period, and by requiring daily intervals, 83 valid, complete data points were available. Using these data, we correlated daily blood cell changes individually in univariate, or jointly in a partial least squares regression, with those predictors that achieved more than $95 \%$ probability density in the positive or negative domain in the PBSC data regression. For each of these predictors, we present the sign of slopes and Bonferroni corrected $p$-values from individual linear regressions.

Joint analysis of the effect of antibiotics and white blood cell counts on the microbiota and the microbiota and immunomodulatory medications on white blood cell counts

743 Analogous to stage 1, we performed cross-validated, regularized linear regressions (ElasticNet) using

744 the scikit-learn package for the Python programming language to jointly estimate the association network between microbiota and circulatory white blood cells. For this, we constructed a block matrix

$\mathbf{X}$ of predictor matrices $\boldsymbol{X}_{\boldsymbol{i}}$ that include the absolute bacterial abundances, drug data (antibiotics for bacterial dynamics and immune modulators for white blood cell dynamics), as well as the counts of white blood cells and a separate intercept term per block. Each block $\boldsymbol{X}_{n_{l}, p_{l}}^{l}$, with $n_{l}$ observations and $p_{l}$ predictors $(1=0 \ldots \mathrm{k})$, on the diagonal of $\mathbf{X}$ corresponds to the indices of the observed daily log-changes of one of the 41 bacterial genera considered in our main analysis or the log changes in neutrophil, lymphocyte and monocyte counts from PBSC patients contained in $\mathbf{Y}$ (in total we calculated 15,833 rates from 256 patients). Our regression problem can thus be written as:

$$
\operatorname{argmin}_{\beta}(\mathbf{Y}-\mathbf{X} \boldsymbol{\beta}) \text { where } \mathbf{X}=\begin{array}{ccc}
\boldsymbol{X}_{\boldsymbol{n}_{0}, p_{0}}^{\mathbf{0}} & \cdots & \mathbf{0}_{\boldsymbol{n}_{0}, \boldsymbol{p}_{\boldsymbol{k}}} \\
\vdots & \ddots & \vdots \\
\mathbf{0}_{\boldsymbol{n}_{\boldsymbol{k}}, \boldsymbol{p}_{0}} & \cdots & \boldsymbol{X}_{\boldsymbol{n}_{\boldsymbol{k}}, \boldsymbol{p}_{\boldsymbol{k}}}^{k}
\end{array}
$$

754 with $\mathrm{k}=44$, i.e. 41 bacterial genera and 3 white blood cell types, the to-be estimated coefficient vector 
in stage 1, elastic net regression, for feature selection. Predictors were scaled between zero and 1, and we used 3-fold cross validation, leaving out $1 / 3^{\text {rd }}$ of the patients at each iteration to identify a global regularization strength, $\lambda$, solving for

$$
\operatorname{argmin}_{\beta}\left\{\frac{1}{2 \eta} \sum_{i=1}^{\eta}\left(y_{i}-\sum_{j=1}^{\rho} x_{i j} \beta_{j}\right)^{2}+\frac{1}{2} \lambda \sum_{j=1}^{\rho}\left|\beta_{j}\right|+\frac{1}{2} \lambda \sum_{j=1}^{\rho} \beta_{j}^{2}\right\}
$$

760

where $\eta$ is the total number of observed daily log changes in genera and white blood cells, and $\rho$ the total number of predictors. This yielded a strongly regularizing $\lambda_{s}$, and thus few predictors. To characterize potential bidirectional relationships between white blood cell counts and the gut microbiota, we iteratively reduced the regularization strength until the strongest interaction between microbiota and white blood cell dynamics, i.e. Faecalibacterium with neutrophil dynamics, was detected. We than re-ran the regression with this reduced regularization strength, $\lambda_{r}$.

\section{Shotgun sequencing}

Sequencing of 124 post-neutrophil engraftment was conducted on the Illumina HiSeq platform. For details and the processing of the FASTQ files, see supplementary methods. We used the HUMAnN2 pipeline ${ }^{70}$ with default settings for functional profiling of our samples, with the UniRef90 data base and ChocoPhlAn for alignment, and we renormalized our samples by library depth to copies per million. We used MetaCyc to obtain stratified and unstratified pathway abundances.

\section{Statistical analysis of shotgun data}

We calculated the predicted microbiota potency score for each sample and separately for neutrophils, lymphocytes and monocytes, by multiplying the abundances of taxa in each of the 124 samples with the corresponding posterior coefficients obtained from the PBSC inference. To distinguish the sets of metabolic functions that separate samples with positive and negative predicted potencies, we converted the pathway abundances into presence and absences profiles. We performed a linear discriminant analysis between positive and negative potency samples with a least squares solver and automatic shrinkage using the Ledoit-Wolf lemma using the sklearn package for the Python programming language ${ }^{67}$. To assess differences in the presence or absence of pathways between samples with positive and negative potency, we used Fisher's exact test for each pathway. 

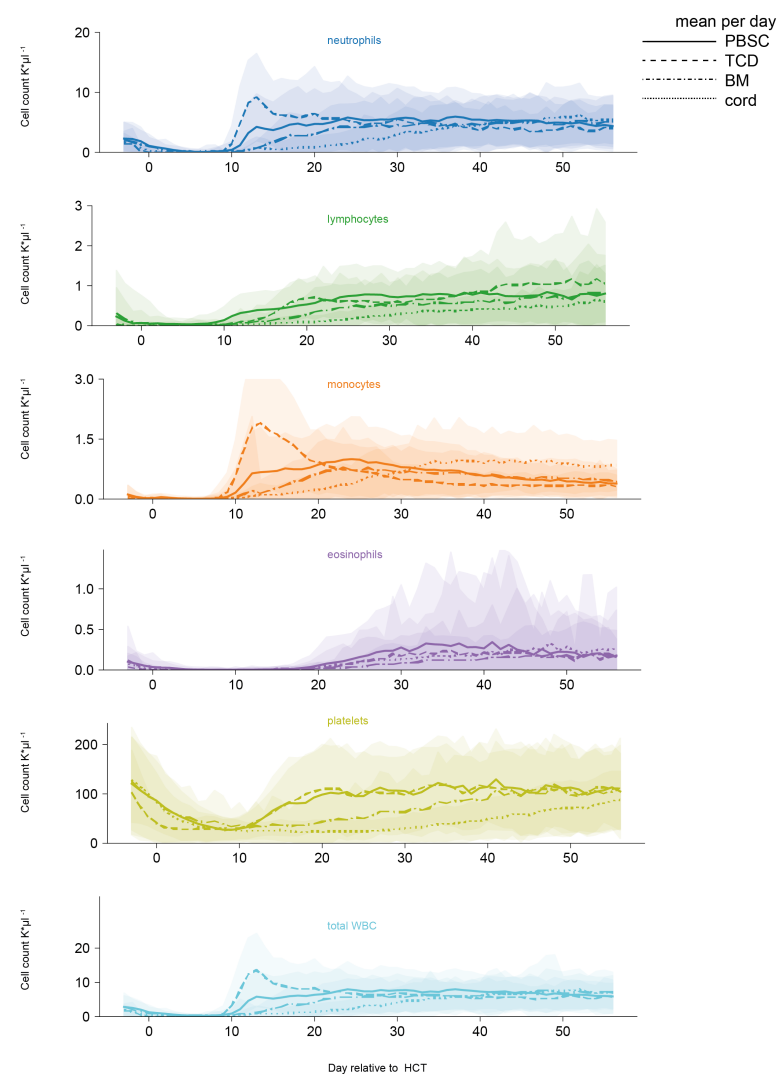

Figure S1: White blood cell counts and platelet counts per graft source over the first 100 days post HCT per day relative to HCT; lines: mean, shaded: \pm standard deviations). 

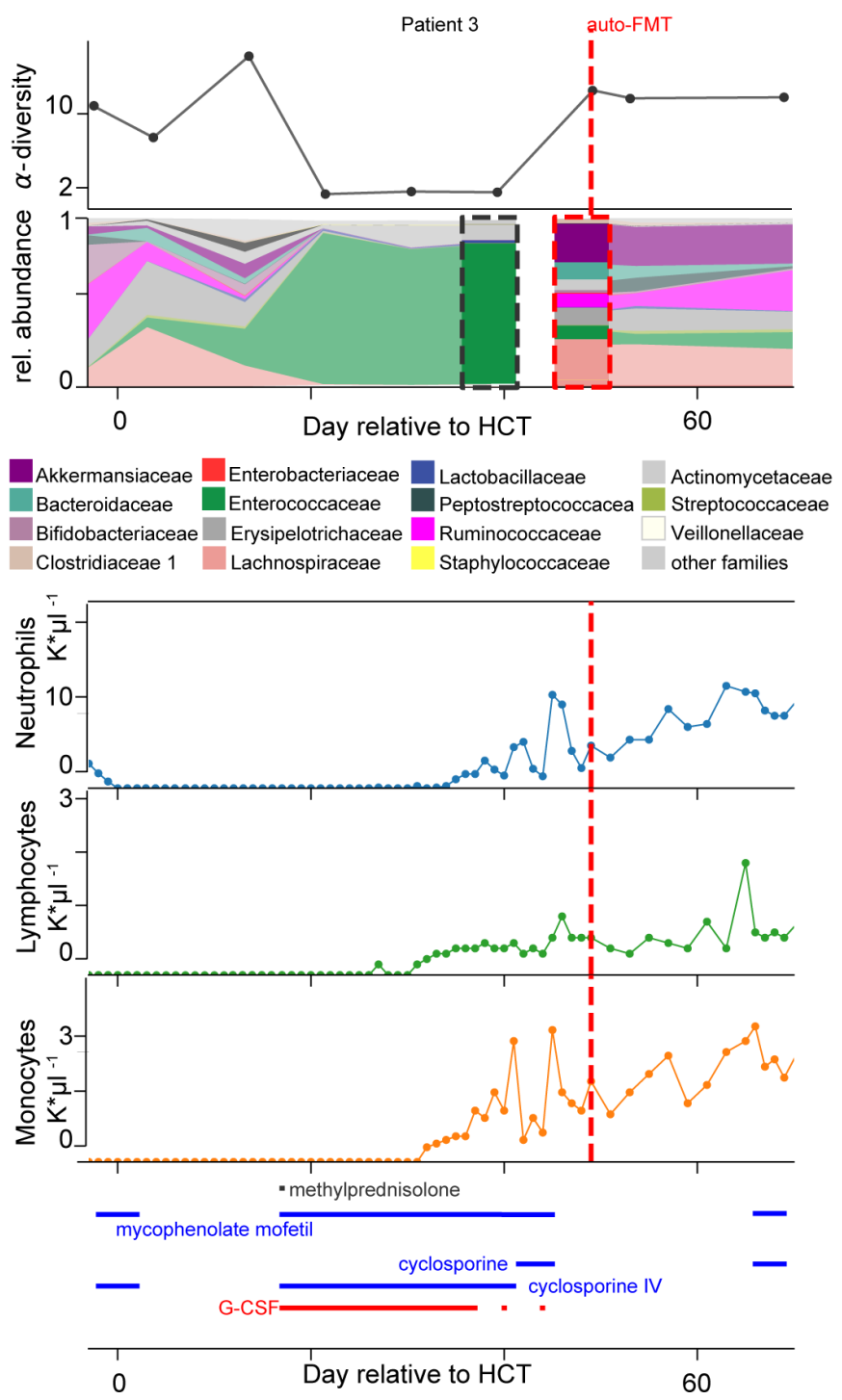

Figure S2: HCT patient who received an autologous fecal microbiota transplant (auto-FMT, dashed red line) that restored commensal microbial families and ecological diversity in the gut microbiota, with concurrent cell counts of peripheral neutrophils, lymphocytes and monocytes and immunomodulatory drug administrations. 
A)

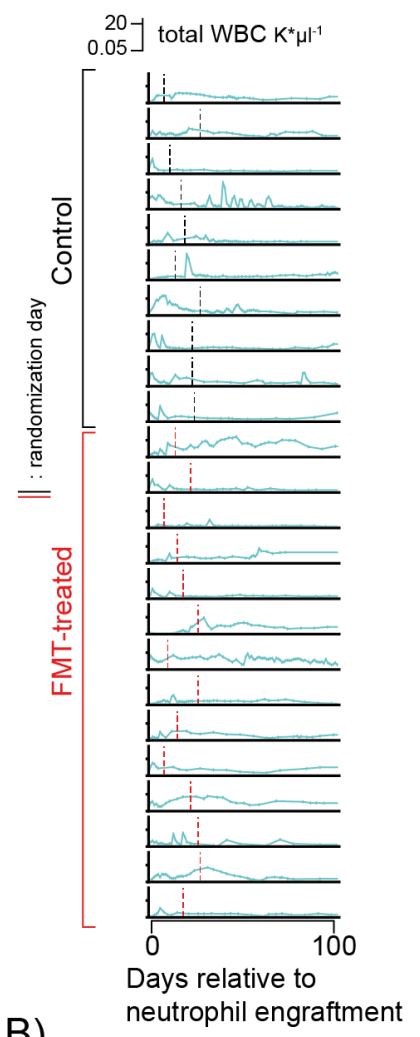

B)

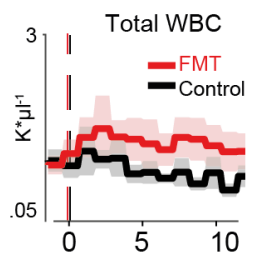

Week rel. to randomization date

C)

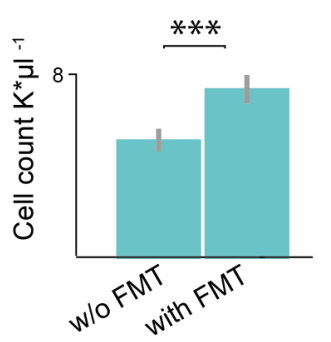

Total WBC

Figure S3: Total counts of white blood cells increased in FMT-treated patients in the weeks enrolled in a randomized controlled trial to receive an autologous fecal microbiota transplant post-neutrophil engraftment (10 control: black vertical line, 14 FMT treated: red vertical line,). B) Weekly mean cell counts aligned to the date of randomization into FMT treatment arm (red) or control (black). Line: weekly mean, shaded region: 95\%-CI C) Results from a linear mixed effects model with random effects per patient and per day relative to neutrophil engraftment confirms that the total white blood cell counts is higher in patients receiving auto-FMT after their treatment as compared to control patients after the randomization date, bars and confidence intervals for the averages of observed white blood cell counts without FMT and post-FMT $(* * *$ : $\mathrm{p}<0.001)$. 


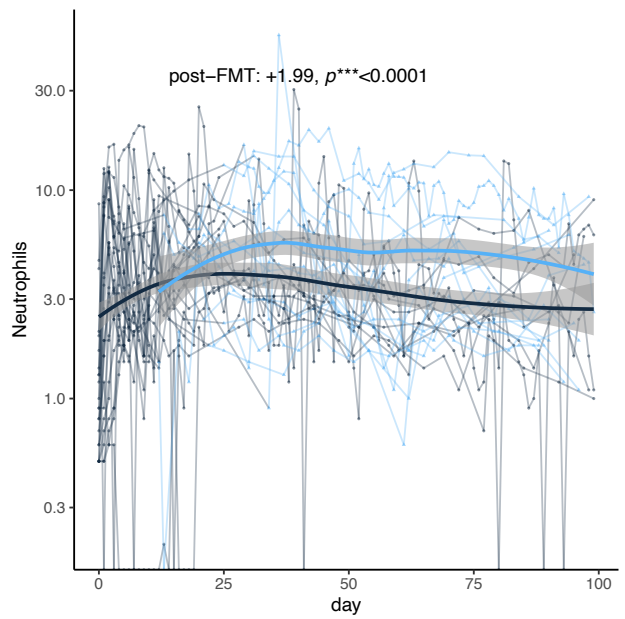

Figure S4: Neutrophil counts in 24. FMT trial patients. Thin lines: raw data (blue: post-FMT); thick black: mean per day, thick blue: mean+post-FMT coefficient. Means and confidence intervals (shaded region) from 814 linear mixed effects model (methods).

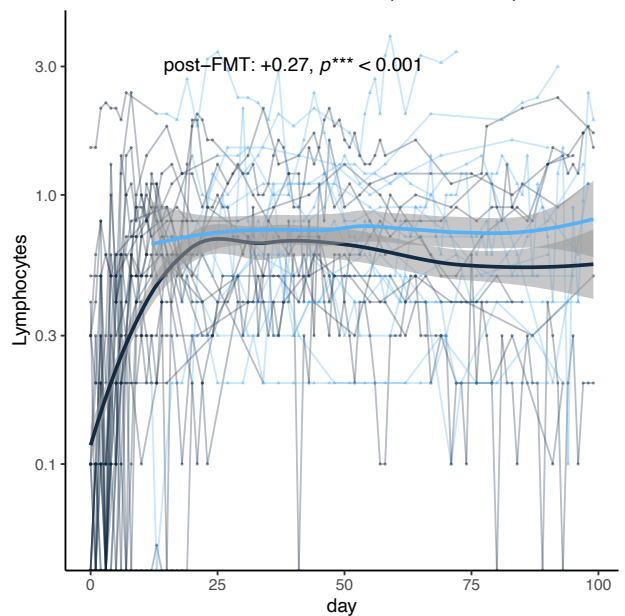

Figure S5: Lymphocyte counts in 24. FMT trial patients. Thin lines: raw data (blue: post-FMT); thick black: mean per day, thick blue: mean+post-FMT coefficient. Means and confidence intervals (shaded region) from linear mixed effects model (methods).

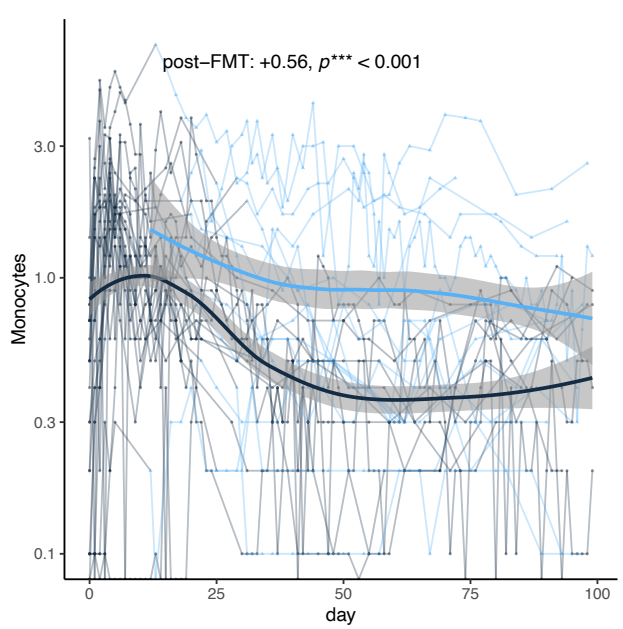

Figure S6: Monocyte counts in 24. FMT trial patients. Thin lines: raw data (blue: post-FMT); thick black: mean per day, thick blue: mean+post-FMT coefficient. Means and confidence intervals (shaded region) from linear mixed effects model (methods). 


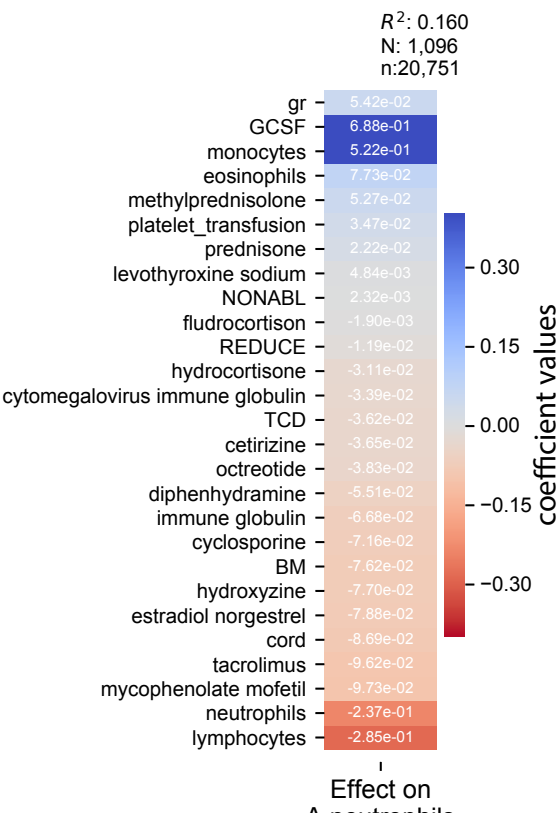

$\Delta$ neutrophils

Figure S7: "Stage 1" regression on neutrophil dynamics on patients without microbiome data. Coefficients from 10-fold cross-validated elastic net regression daily changes in neutrophils. gr: intercept; TCD: T-cell depleted graft (ex-vivo) by CD34+selection; PBSC: peripheral blood stem cells; BM: bone marrow; cord: umbilical cord blood; NONABL: Nonmyeloablative; REDUCE: reduced-intensity conditioning regimen; F: female; N: patients, $n$ : samples (daily changes in neutrophils).

\section{Effect on}

$\Delta$ lymphocytes

Figure S8: "Stage 1" regression on lymphocytes dynamics on patients without microbiome data. Coefficients from 10-fold cross-validated elastic net regression daily changes in lymphocytes. gr: intercept; TCD: T-cell depleted graft (ex-vivo) by CD34+selection; PBSC: peripheral blood stem cells; BM: bone marrow; cord: umbilical cord blood REDUCE: reduced-intensity conditioning regimen; F: female. N: patients, n: samples (daily changes in lymphocytes). 


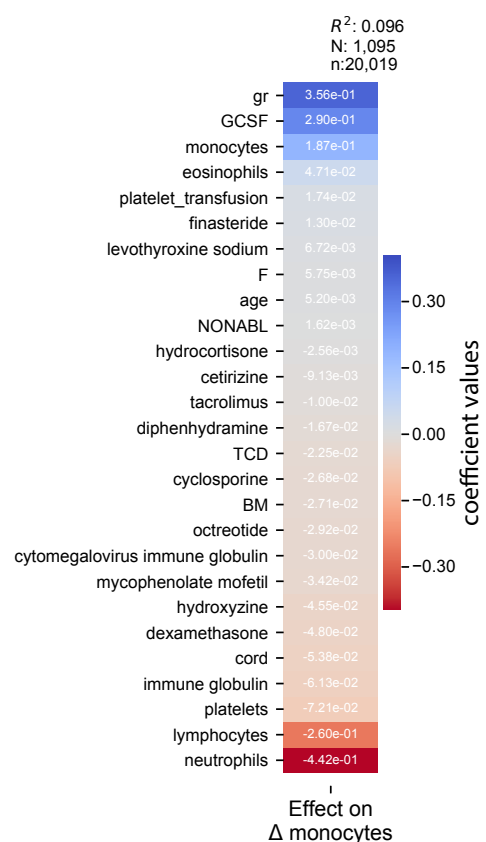

Figure S9: "Stage 1" regression on lymphocytes dynamics on patients without microbiome data. Coefficients from 10-fold cross-validated elastic net regression daily changes in lymphocytes. gr: intercept; TCD: T-cell depleted graft (ex-vivo) by CD34+selection; PBSC: peripheral blood stem cells; BM: bone marrow; cord: umbilical cord blood REDUCE: reduced-intensity conditioning regimen; F: female. N: patients, n: samples (daily changes in lymphocytes). 
A)

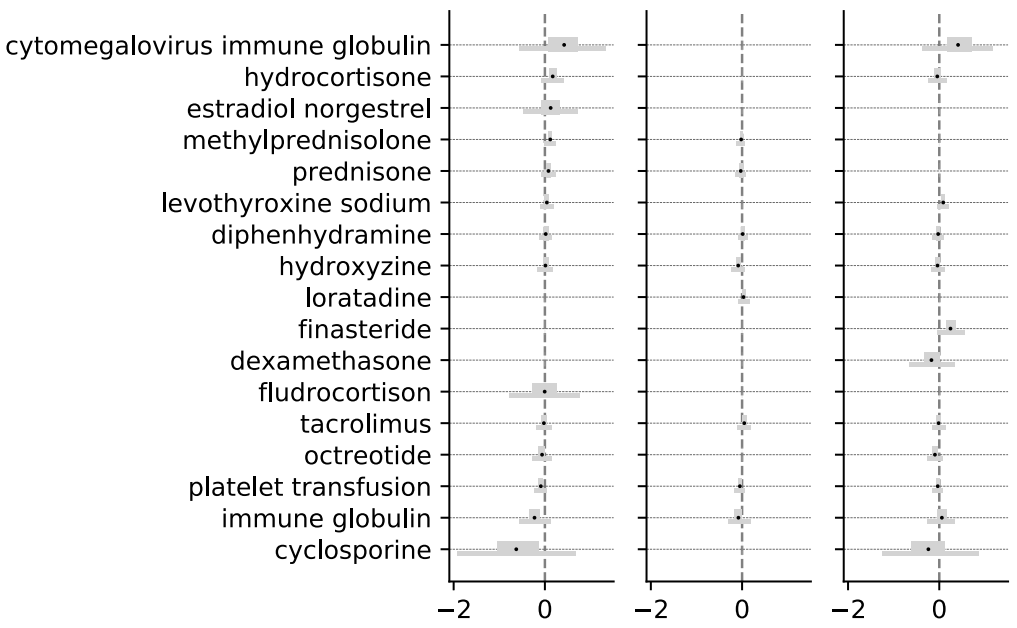

B)

[Eubacterium] coprostanoligenes group

[Ruminococcus] torques group Atopobium

Flavonifractor

Lactobacillus

Actinomyces

Lachnoclostridium

Escherichia-Shigella

Agathobacter

Erysipelatoclostridium

Enterococcus
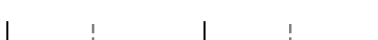

Blautia

unid.Peptostreptococcaceae 9

UBA1819

Subdoligranulum

Bifidobacterium

Anaerostipes

unid. Lachnospiraceae 20

Eggerthella

Sellimonas

Lactococcus

Ruminiclostridium 5

Mogibacterium

Gemella

Intestinibacter

Streptococcus

Parvimonas

Scardovia

C)

Figure S10: Additional coefficient estimates of medications (A), additional genera (B) and metadata (C) from the Bayesian regression, see also Figure 3. REDUCE: reduced-intensity conditioning regimen; NONABL: nonmyeloablative conditioning regimen. F: female
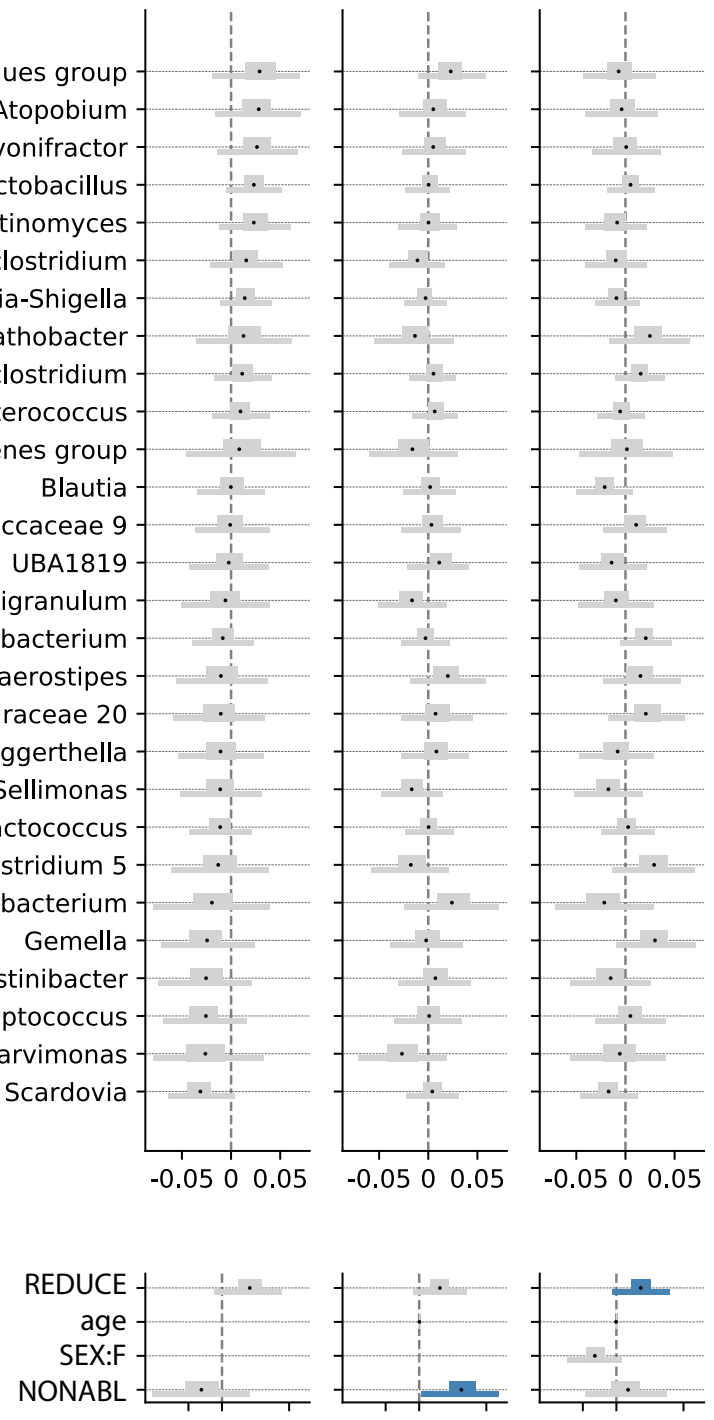


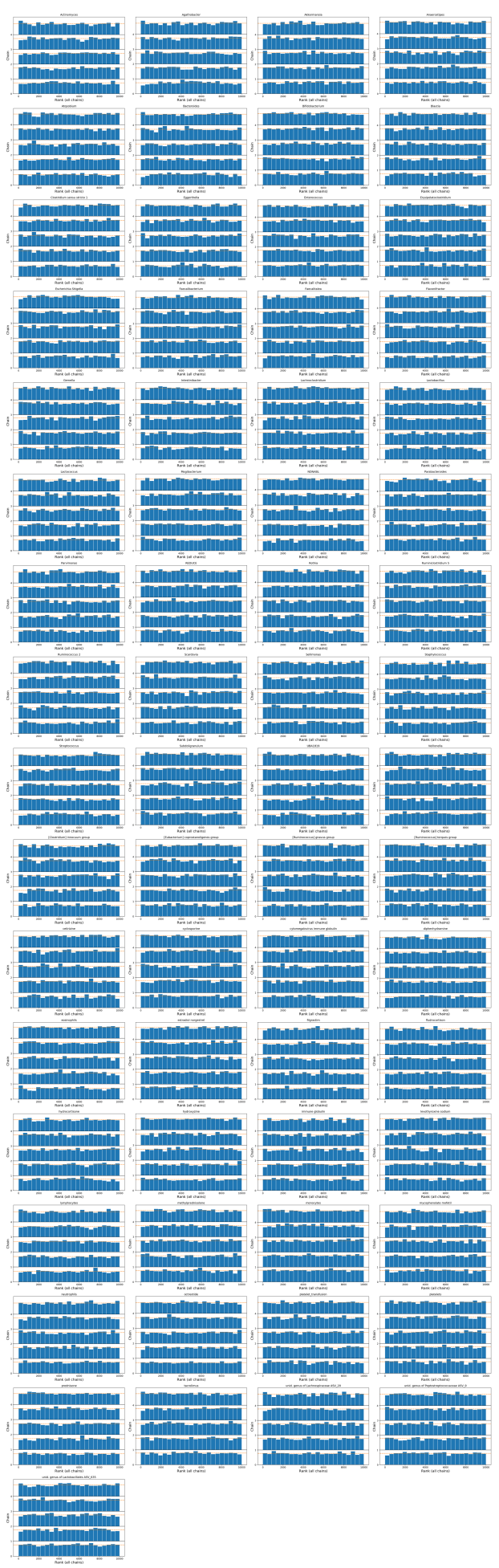

Figure S11: Posterior sampling converged. Histograms of the ranked posterior draws from the model of neutrophil dynamics in PBSC patients (ranked over all chains), plotted separately for each chain (see supplementary methods), show no substantial differences between chains ${ }^{71}$. 

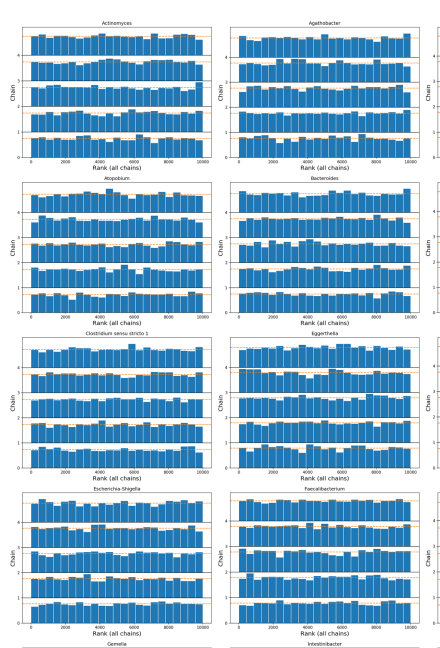

=
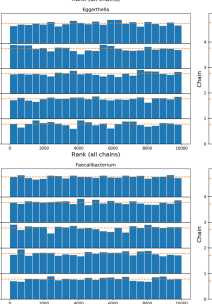

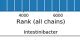

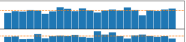

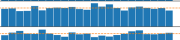

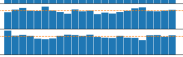

m|

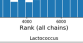

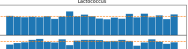

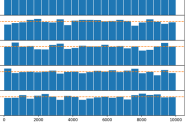

-1

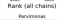

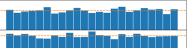

m|

-

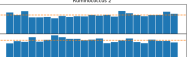

(17)

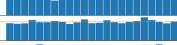

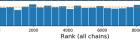

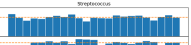

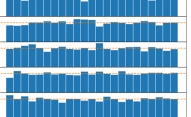

(1)

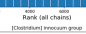

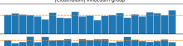

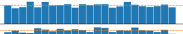

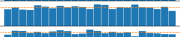

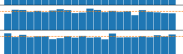

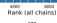

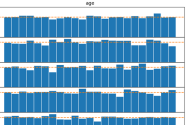

(1)

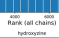

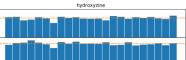

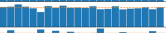

(1)

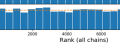

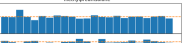

1)

Hin

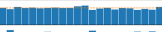

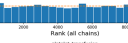

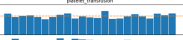

HIn

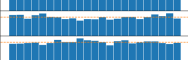

(1)

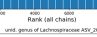

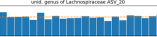

(10,

s

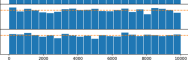

855

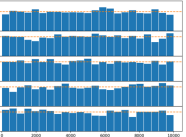

sinturenes

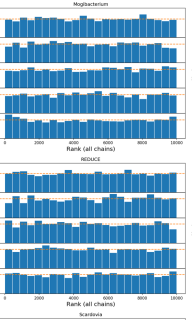

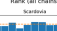

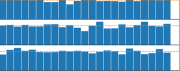

(1)

(1)

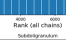

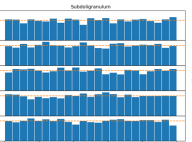

Sintercenter

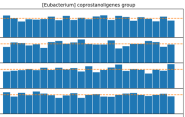

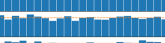

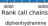

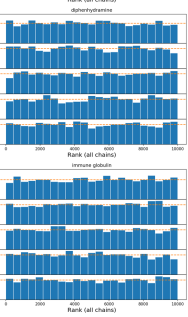

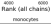

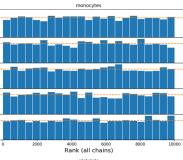

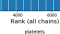

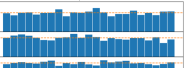

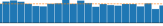

nIm

매

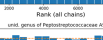

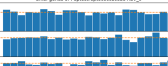

(110)

nen
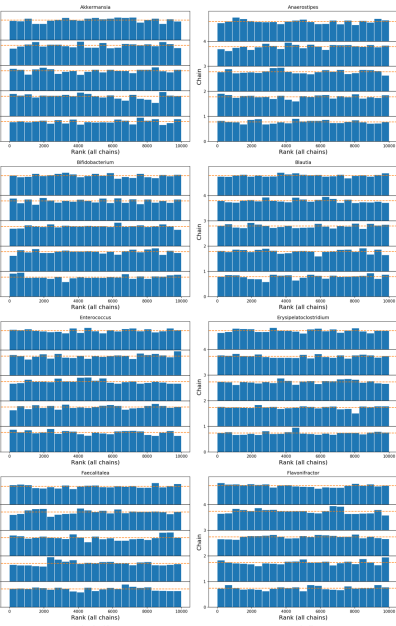

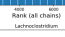

|

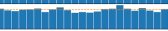

$=-1$

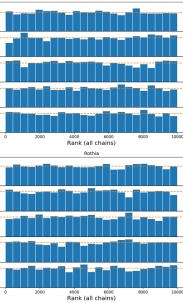

nnthen

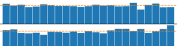

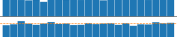

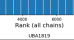

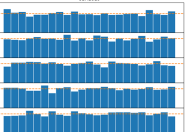

Eintarasonis

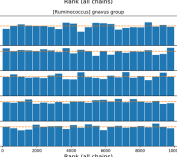

Lintatcoson

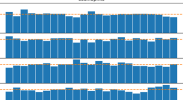

(1)

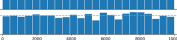

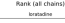

||

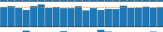

(1)

siturats

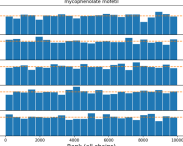

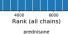

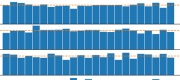

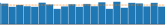

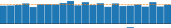

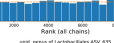

non

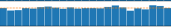

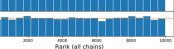

Figure S12: Posterior sampling converged.

Histograms of the ranked posterior draws from the model of lymphocyte dynamics in PBSC patients (ranked over all chains), plotted separately for each chain (see supplementary methods), show no substantial differences between chains ${ }^{71}$. 
Figure S13: Posterior sampling converged. Histograms of the ranked posterior draws from the model of monocyte dynamics in PBSC patients (ranked over all chains), plotted separately for each chain (see supplementary methods), show no substantial differences between chains ${ }^{71}$.
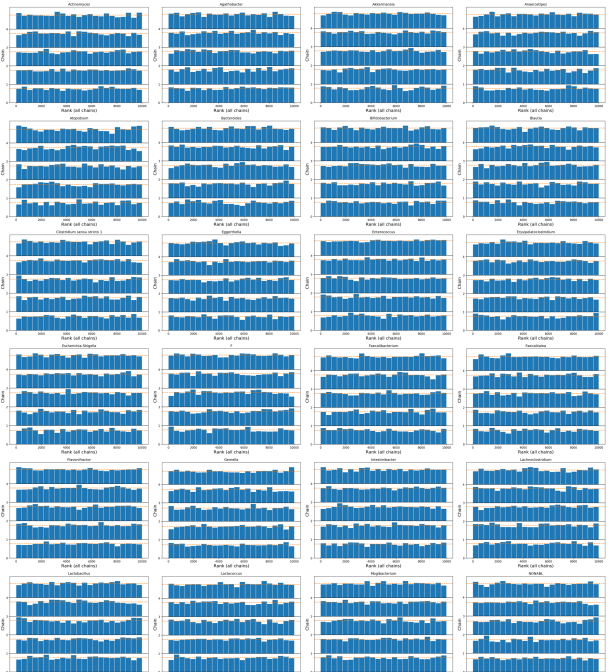

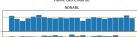

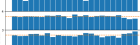

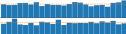

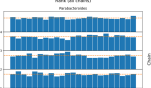

-

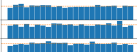

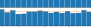

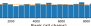

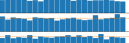

nnd

-

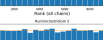

(I)

minntrinter

ing

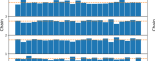

(1)

nimentint

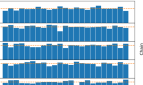

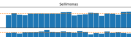

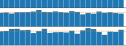

1)

(n)

(1)

minter
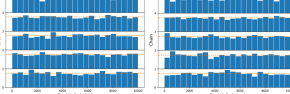

(n)

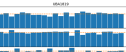

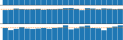

(n)

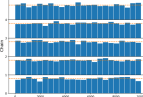

nury

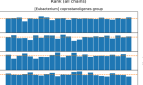

110

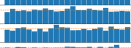

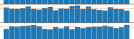

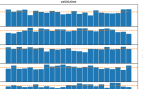

-

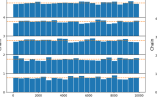

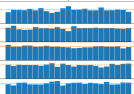

(1)

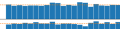

(1)

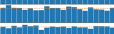

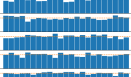

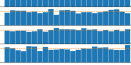

thent

mint

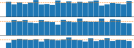

minn?

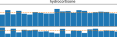

,

(1)

(1)

115

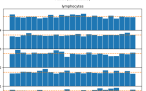

nund

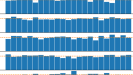

(I)

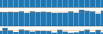

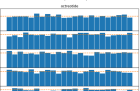

ntwilnum

$+$
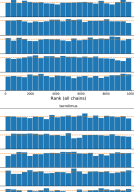

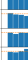

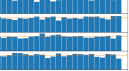

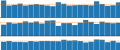

(1)

Inn = 

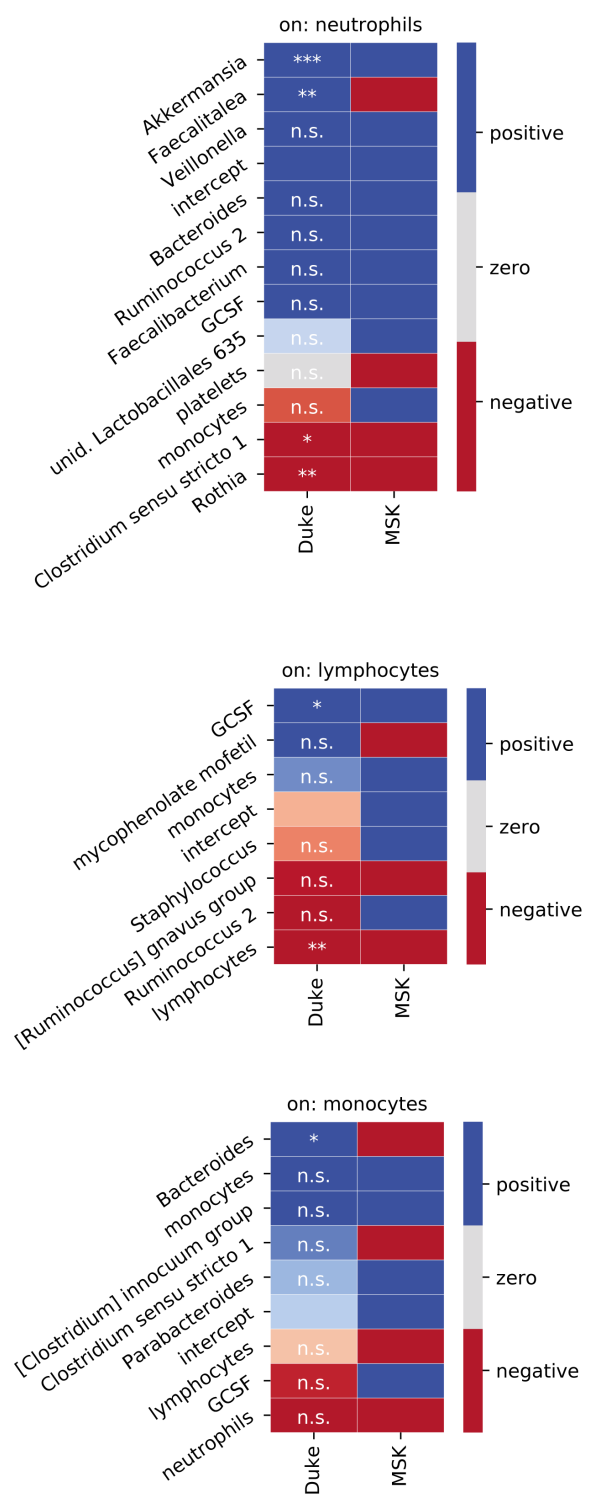

Figure S14: Validation analysis of predictors on white blood cell dynamics using data from patients treated at DukeHealth. Individual univariate regressions of microbiome and clinical predictors identified in stage 2 of our analysis on daily changes in neutrophils, lymphocytes and monocyte. Bonferroni corrected p-values: $* * *<0.001$, $* *<0.01, *<0.05 ; \mathrm{p}>0.05$ : n.s. Sign of coefficients from MSK PBSC patients for comparison. 


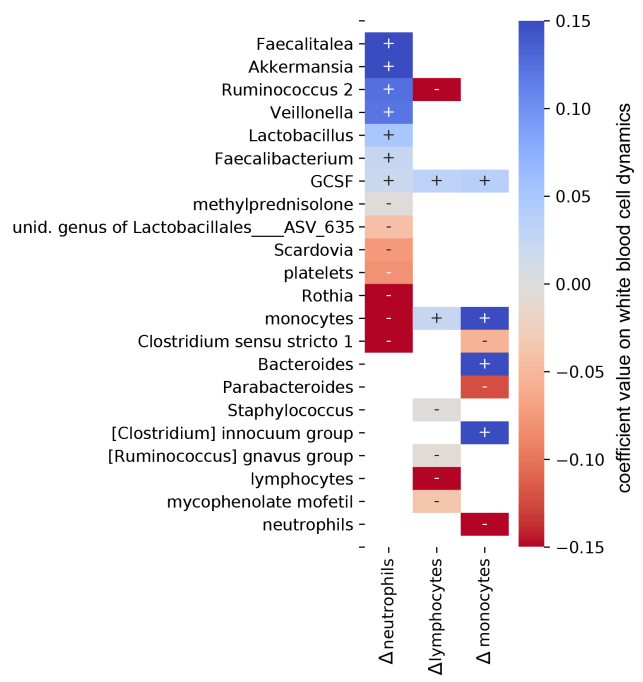

Figure S15: Validation analysis of predictors on white blood cell dynamics using data from patients treated at DukeHealth. Partial least squares regression of microbiome and clinical predictors identified in stage 2 of our analysis on daily changes in neutrophils, lymphocytes and monocyte.

A) n:389, N:120 n:331, N:109 n:376, N:120

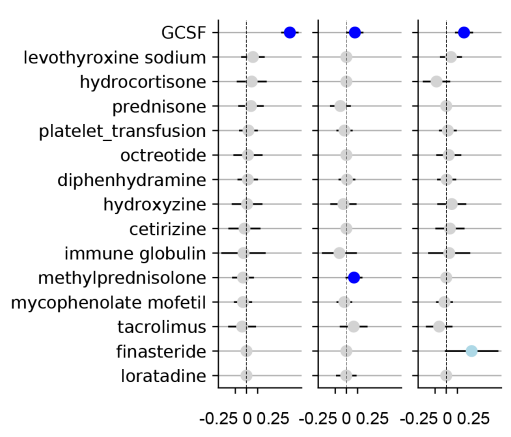

B)

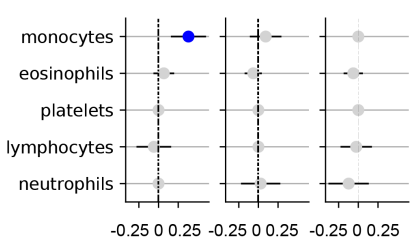

C)

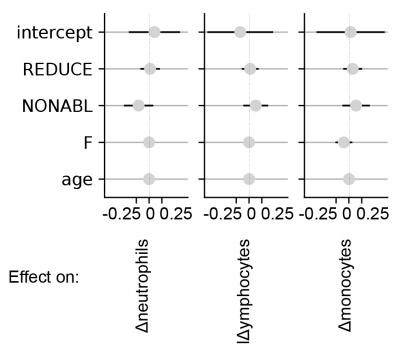

D)

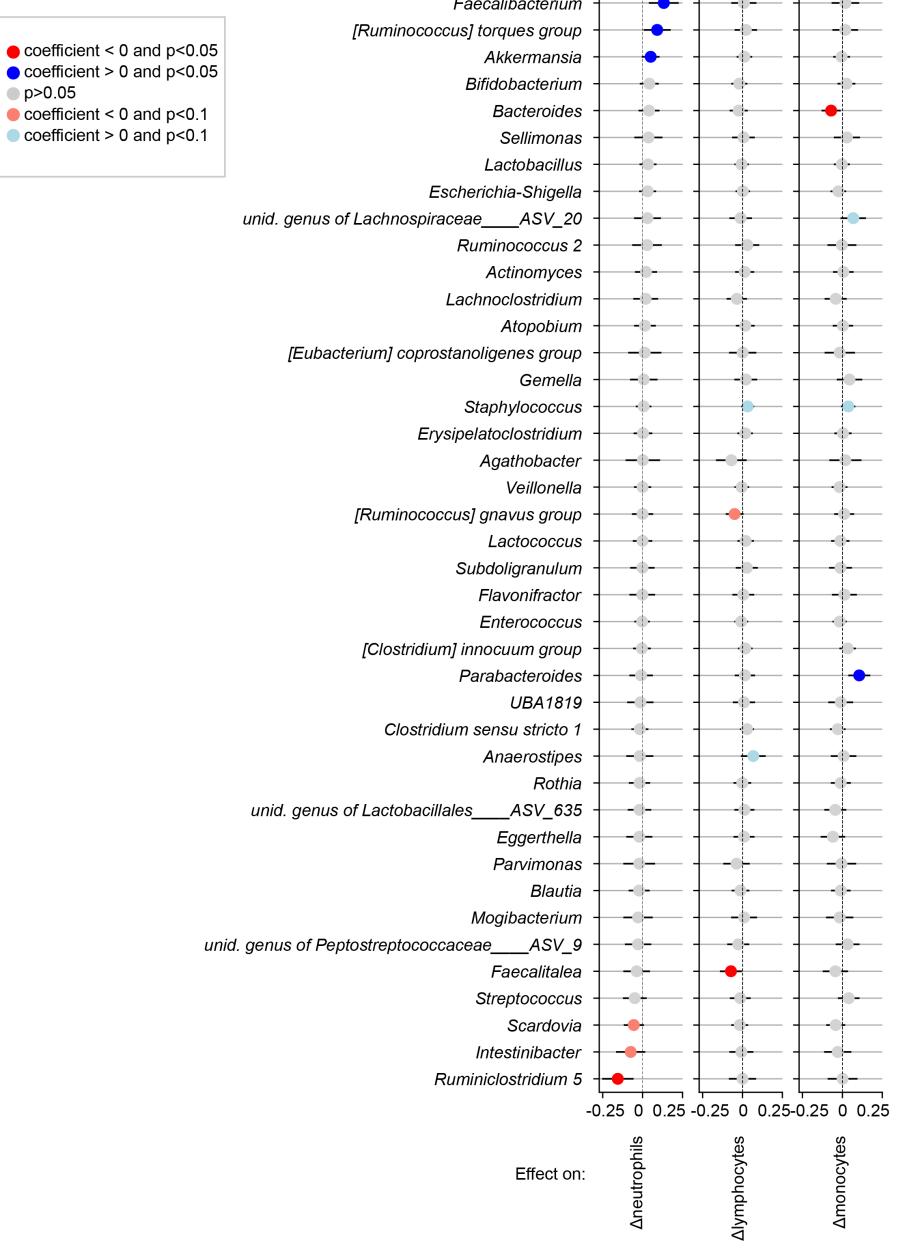


Figure S16: Validation analysis of the main model using absolute bacterial abundances as predictors instead of relative abundances in Figure 3. Results show coefficients from a least squares regression for medications (A), white blood cell feedbacks (B) metadata (C) and total genus abundances (D) of neutrophil, lymphocyte and monocyte daily log-changes. This was only possible for only a subset of the data for which we obtained absolute bacterial abundance estimates (methods), n: samples, N: patients.
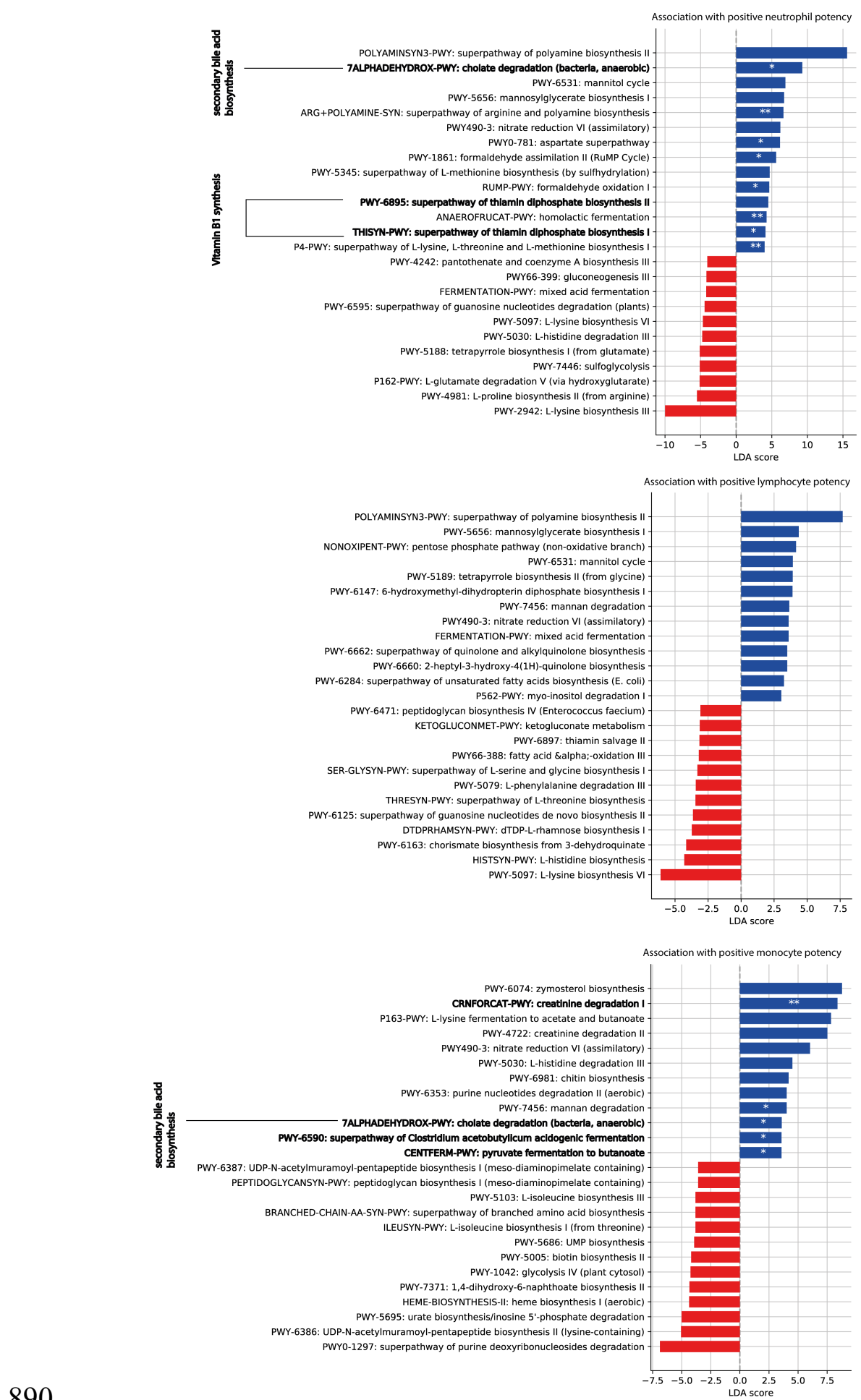

Figure S17: Functional analysis of microbiota samples. To distinguish samples predicted to increase rates of 
pathway profiles from 124 shotgun sequenced samples that distinguished positive and negative potency samples the most (LDA-score magnitude in the $95^{\text {th }}$ percentile). Highlighted pathways are discussed in the main text. For each pathway, we tested differences between positive and negative potency samples using Fisher's exact test; pvalue $<0.001: * * *,<0.01: * *,<0.05: *$.

902

903

904

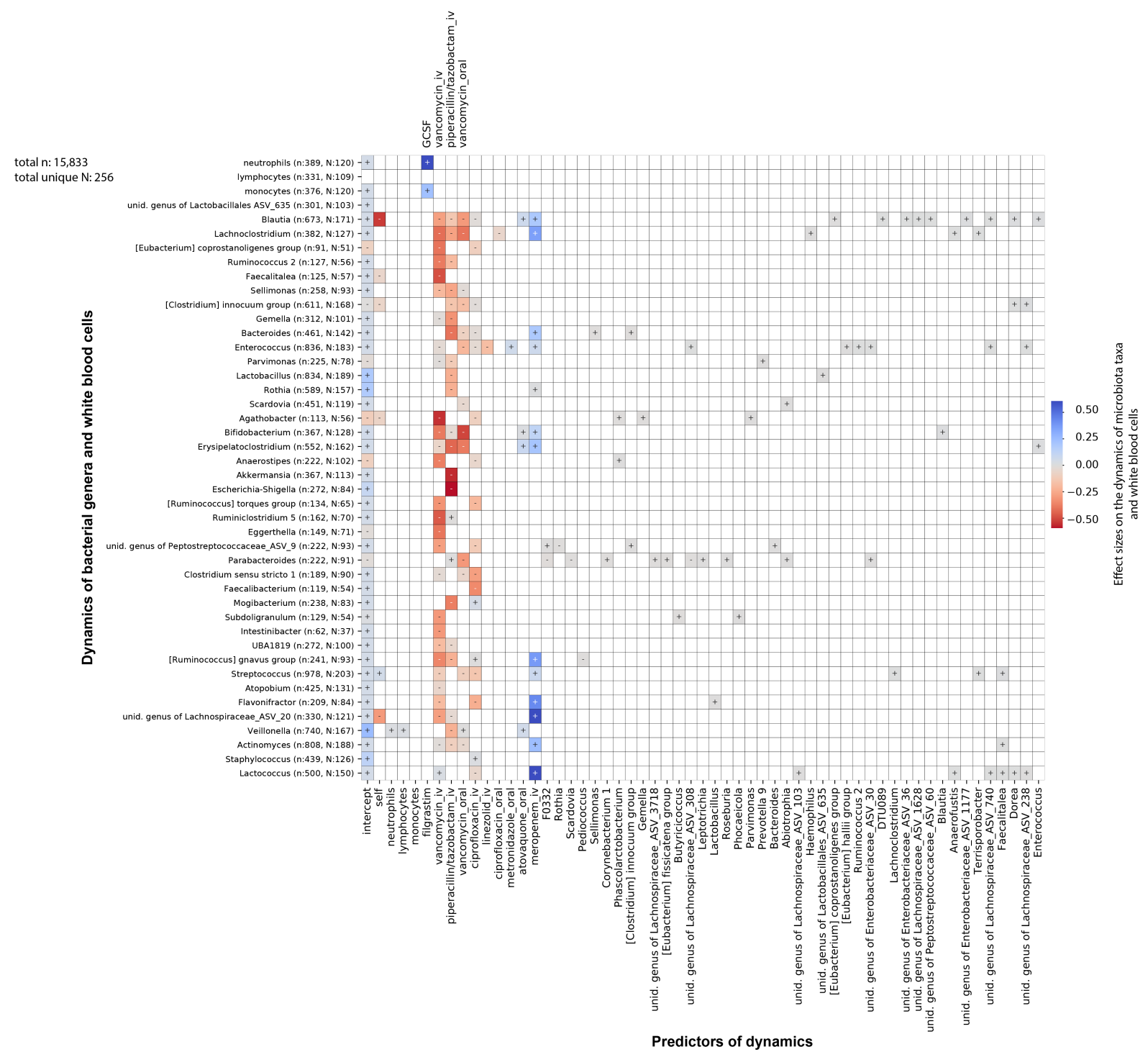

Figure S18: Jointly inferred association network between white blood cell and bacterial genus dynamics 


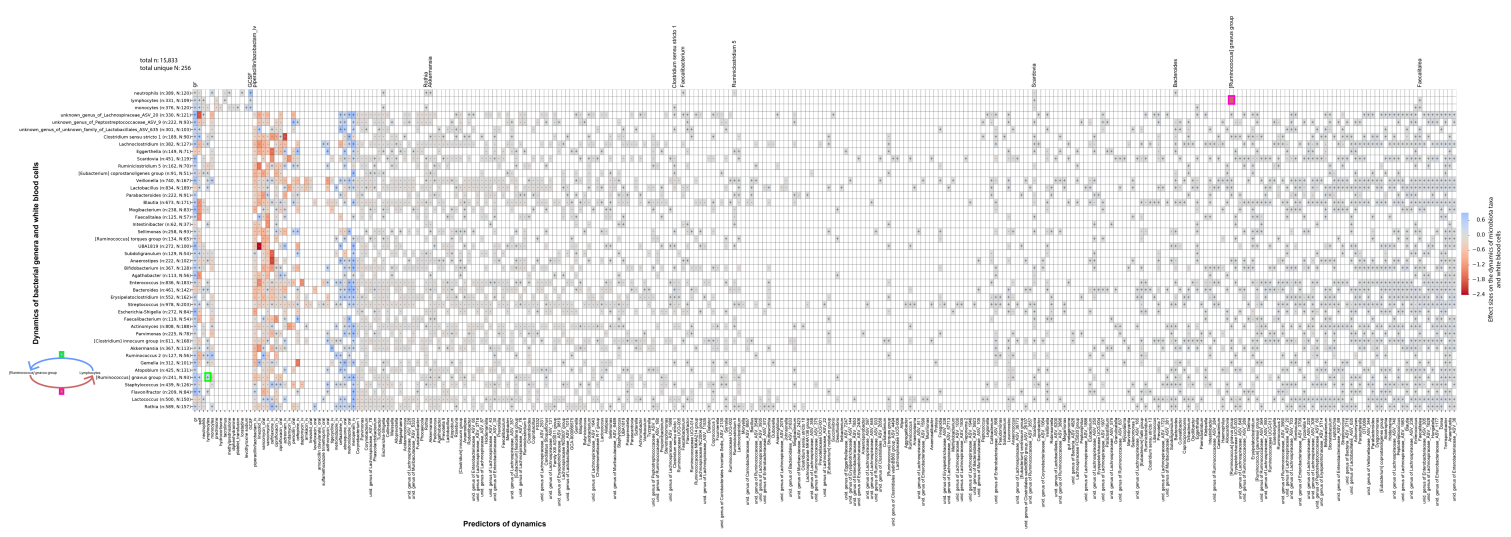

Figure S19: Jointly inferred association network between white blood cell and bacterial genus dynamics with reduced regularization (methods) indicates potential bidirectional feedbacks, e.g. between lymphocytes and [Ruminococcus] gnavus group (highlighter green boxes, and cartoon).

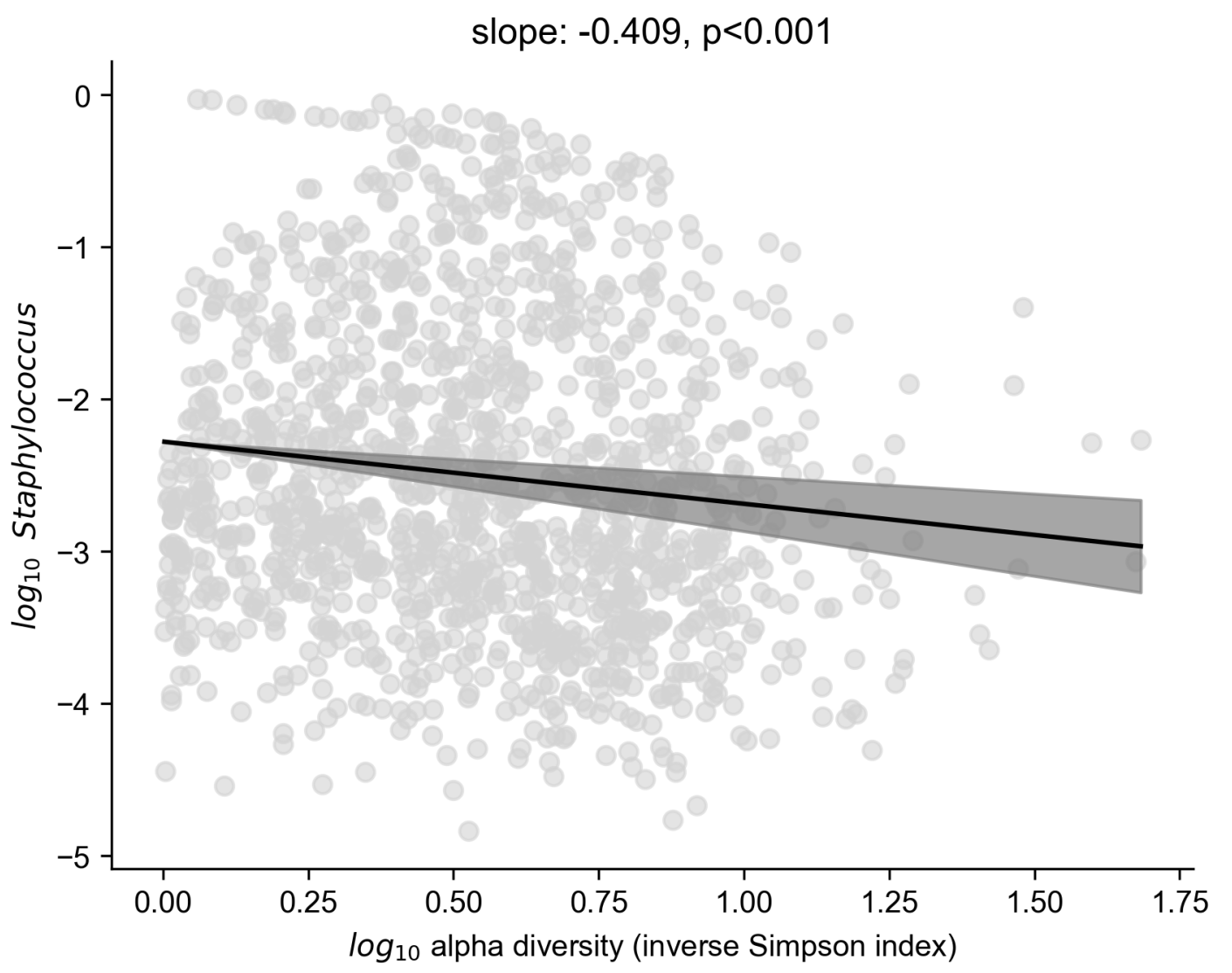

Figure S20: The relative non-zero abundance of Staphylococcus is inversely related to microbiome alpha diversity, shaded: $95 \%$ confidence intervals. 

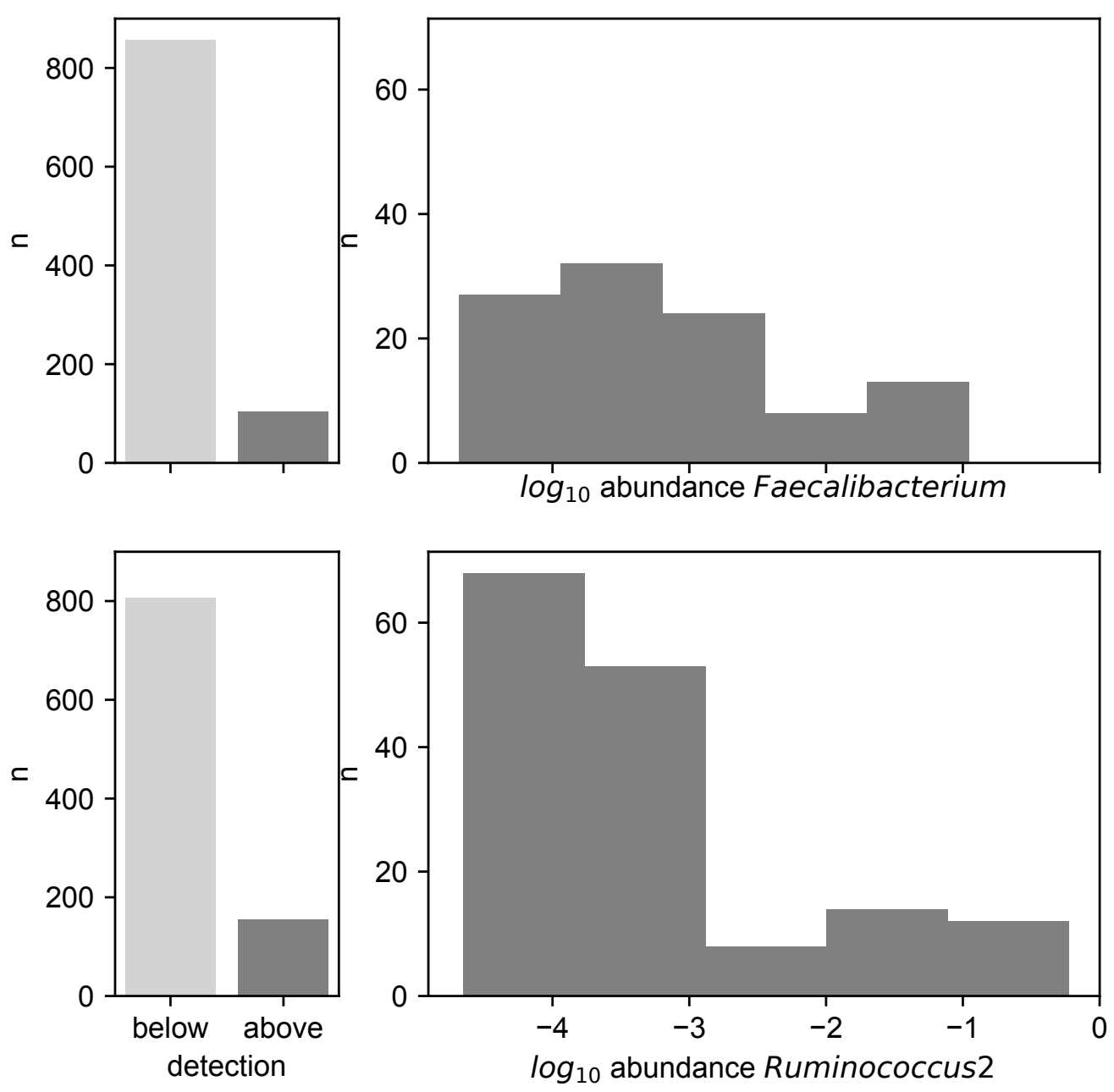

Figure S21: Abundance profiles of the two genera, Faecalibacterium and Ruminococcus 2, most strongly associated with white blood cell increase; number of times detected (left) and $\log _{10}$ abundance distribution when above detection (right).
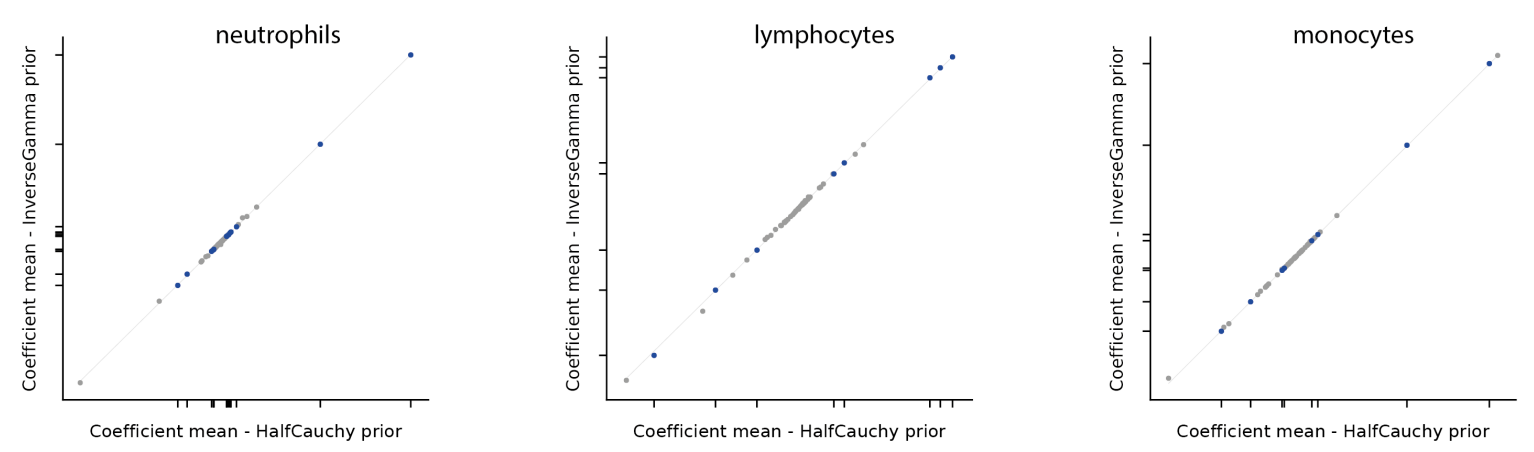

Figure S22: Posterior association coefficients do not depend on the choice of prior for $\sigma$ in the main Bayesian model. Plotted are the posterior means from our main analysis against the equivalent inference with an inverse Gamma prior (alpha $=1$, beta $=1$ ). 
Table S1: Data set summary and patient characteristics. HCT-graft types: TCD: T-cell depleted graft (ex-vivo) by CD34+selection; PBSC: peripheral blood stem cells; BM: bone marrow; cord: umbilical cord blood; Conditioning intensity: Bacigalupo classification, graded categories from most to least intense (ABLATIVE,

945 REDUCE, NONABL).

946

\section{patients \\ HCT therapies* \\ blood samples}

Disease

\section{HCT graft type}

\section{Conditioning intensity}

\section{Gender}

Age of adults (years)

\section{Microbiome samples}

*) some patient received several HCTs

Table S2: Patient and HCT characteristics of 24 patients enrolled in the randomized controlled FMT trial.

\begin{tabular}{|l|r|r|}
\hline & control & FMT treated \\
\hline N patients & 10 & 14 \\
\hline ABLATIVE & 6 & 7 \\
\hline REDUCE & 4 & 7 \\
\hline BM unmodified & 1 & 3 \\
\hline PBSC unmodified & 3 & 4 \\
\hline TCD & 5 & 3 \\
\hline cord & 1 & 4 \\
\hline
\end{tabular}

total

between HCT-day -21 and HCT-day 183

Leukemia

Non-Hodgkin's Lymphoma

Multiple Myeloma

Hodgkin's disease

other

PBSC unmodified

BM unmodified

cord

NONABL

$\mathrm{M}$

$25 \%$-tile

$75 \%$-tile

total

patients with microbiome sample 
959 Table S3: Patient and HCT characteristics of the subset of patients who donated microbiota samples.

patients

Disease distribution

HCT graft type

\section{Conditioning intensity}

Gender

Age (years)
Leukemia

Non-Hodgkin's Lymphoma

Multiple Myeloma

Hodgkin's disease

other

TCD

PBSC unmodified

BM unmodified

cord

ABLATIVE

REDUCE

NONABL

$\mathrm{M}$

F

25\%-tile

mean

$75 \%$-tile

$51 \%$

$15 \%$

$8 \%$

$3 \%$

$23 \%$

$37 \%$

$38 \%$

$9 \%$

$16 \%$

$55 \%$

$34 \%$

$11 \%$

$59 \%$

$41 \%$

46

54

65

960

961

962

Table S4: Patient and HCT characteristics of the subset of patients who did not donate microbiota samples.

patients

Disease distribution

\section{HCT graft type}

\section{Conditioning intensity}

\section{Gender}

Age (years)
Leukemia

Non-Hodgkin's Lymphoma

Multiple Myeloma

Hodgkin's disease

other

TCD

PBSC unmodified

BM unmodified

cord

ABLATIVE

REDUCE

NONABL

$\mathrm{M}$

F

$25 \%$-tile

mean

$75 \%$-tile
$53 \%$

$17 \%$

$5 \%$

$5 \%$

$20 \%$

$40 \% \%$

$31 \%$

$17 \%$

$12 \%$

$65 \%$

$14 \%$

$21 \%$

$58 \%$

$42 \%$

36

47

59
963

964

965

966

Table S5: Patient and HCT characteristics of the Duke University patient cohort.

patients

Disease distribution
Lymphoma

Leukemia

Non-Hodgkin's Lymphoma

Multiple Myeloma 


$\begin{array}{lr}\text { Hodgkin's disease } & 4 \% \\ \text { other } & 24 \% \\ \text { TCD } & 0 \% \\ \text { PBSC unmodified } & 72 \% \\ \text { BM unmodified } & 11 \% \\ \text { cord } & 16 \% \\ \text { ABLATIVE } & 92 \% \\ \text { NONABL } & 7 \% \\ \text { M } & 65 \% \\ \text { F } & 35 \% \\ 25 \% \text {-tile } & 41 \\ \text { mean } & 49 \\ 75 \% \text {-tile } & 57\end{array}$

\section{Data availability}

The data used in our study is organized in supplementary tables (data-tables.zip), with corresponding filenames (italic):

1. cGENUS.csv: relative taxon abundances in fecal microbiota samples from 12,633 stool samples

2. cHCTMETA.csv: HCT characteristics

3. cINFECTIONS.csv: positive blood culture results

4. cMISAMPLES.csv: NCBI SRA accession number, diversity (inverse Simpson index), total $16 \mathrm{~S}$ (where available), stool consistency for each fecal microbiota sample

5. cMED.csv: medication data

6. cPIDMETA.csv: anonymized patient demographics

7. $c W B C . c s v$ : absolute counts of neutrophils, lymphocytes, monocytes, eosinophils, and platelets with indication if included in analyses

8. cDUKE_GENUS.csv: relative taxon abundances in fecal microbiota samples from 12,633 stool samples

9. $c D U K E \_W B C . c s v$ : absolute counts of neutrophils, lymphocytes, monocytes, eosinophils, and platelets with indication if included in analyses

10. cDUKE_MED.csv: medication data

11. cFMT_analysis.csv: convenience table for Figure 2

\section{Code availability}

The relevant scripts for stage 1, stage 2, and the model assessing the effect of FMT on white blood cell counts are on github:https://github.com/jsevo/wbcdynamics_microbiome. 